\title{
Topology of the skyrmion bundle
}

Cite as: Journal of Mathematical Physics 36, 4406 (1995); https://doi.org/10.1063/1.530899 Submitted: 20 December 1994 . Accepted: 22 March 1995 . Published Online: 04 June 1998

\section{Christian Gross}

\section{ARTICLES YOU MAY BE INTERESTED IN}

Solutions of the Einstein and Einstein-Maxwell Equations

Journal of Mathematical Physics 10, 1842 (1969); https://doi.org/10.1063/1.1664769

Topological BF theories in 3 and 4 dimensions

Journal of Mathematical Physics 36, 6137 (1995); https://doi.org/10.1063/1.531238

Cohomology and connections on fiber bundles and applications to field theories Journal of Mathematical Physics 37, 6375 (1996); https://doi.org/10.1063/1.531783

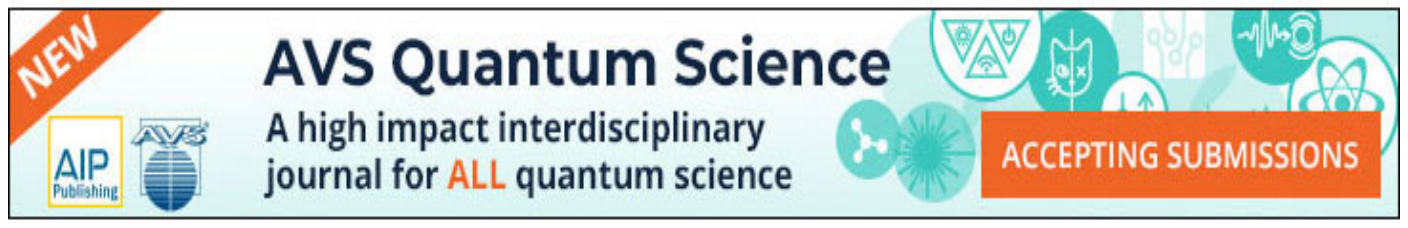




\title{
Topology of the skyrmion bundle
}

\author{
Christian Gross \\ Fachbereich Mathematik, TH Darmstadt, Germany
}

(Received 20 December 1994; accepted for publication 22 March 1995)

We compute homotopy and cohomology groups for the Skyrmion bundle, which play an important role in the description of the monopole-induced proton decay within the Skyrme model. By use of spectral sequences, the differential forms on the bundle that allow for the calculation of baryon numbers and for the anomalous action are developed out of the ones on the fiber, and are adapted to the given Maxwell connection. (c) 1995 American Institute of Physics.

\section{INTRODUCTION}

During the last years many physicists seem to have rediscovered the Skyrme model ${ }^{1}$ in theoretical nuclear physics as an effective field theory related to quantum chromodynamics (QCD) by its underlying symmetry. Yet most of the articles deal with the ungauged, purely hadronic case treating interactions between baryons and mesons and do not cover interactions between these particles and electromagnetic fields, although especially for the latter case, the Skyrme model reveals some interesting features. Grand unification theory in its present form implies that magnetic monopoles $M$ are able to catalyze baryon-number-violating processes like

$$
M+p^{+} \rightarrow M+e^{+}+\text {pions }
$$

and it has been shown ${ }^{2}$ that these processes can conveniently be described within the Skyrme model, even if the hope to proceed to a reaction cross section was in vain. ${ }^{3}$

The correct settings for the purpose of treating interactions with electromagnetic fields are that of a skyrmion bundle (and a lepton bundle) associated with a principal $U_{1}$ bundle and a Maxwell connection on it, and there still seems to be a need for clarifying the foundations of the skyrmion bundle. The possibility of describing baryonic processes by means of the mesonic fields alone, is essentially based on the topological properties of the unitary groups $\mathrm{SU}_{m}$. While their stable homotopy groups and their de-Rham cohomology including the generators $\omega_{2 n+1}$ of $H^{2 n+1}\left(\mathrm{SU}_{m}\right)$ are well known in the literature, ${ }^{4,5}$ little is noted on the topology of the skyrmion bundle, although this is quite indispensable. In order to treat baryon-number-violating processes, one needs an analog of the (normalized) differential form $\omega_{3}$, which counts the number of baryons described by a certain mesonic field configuration. One also needs an analog of $\omega_{5}$, which serves as a base for the anomalous action.

Several approaches ${ }^{6-8}$ have been made to generalize $\omega_{3}$ and $\omega_{5}$ to the bundle case. Yet all these approaches were local ones and thus could only prove closedness and gauge invariance of the recovered forms, but they could not answer the question of whether these forms are exact or generate non-trivial cohomology groups. Instead, we will choose a different approach and examine homotopy and cohomology of the skyrmion bundle in general. For the purpose of recovering (global) differential forms on the bundle from those on the fiber $\mathrm{SU}_{m}$, we will use the quite intricate technique of spectral sequences. In a second step we will then gauge these differential forms by adapting them to the given Maxwell connection.

For notational convenience and as a foundation, the next section gives a survey on the ungauged Skyrme model with respect to its topological properties. We introduce the invariant currents and state the index theorem that is responsible for all topological quantizations within the Skyrme model. In addition, we present the Hodge star operator and the co-derivation of differen- 
tial forms, which are important not only for the definition of a conserved baryonic current, but also for treating electromagnetism by means of differential geometry.

Section III summarizes this approach and introduces fiber bundles and differential geometry on them. For our purpose, we concentrate on the case of a one-dimensional Lie algebra $\mathfrak{g} \cong \mathbf{R}$.

In section IV we apply the results to the monopole bundle as a principal $U_{1}$ bundle and the skyrmion bundle associated to it. Especially, the prototype for the interaction between magnetic monopoles and skyrmions, the bundle for the single monopole, is treated and completely classified by its magnetic charge. We give the transformation rules for the locally defined forms and their projections induced by the Maxwell connection.

After a survey over the homotopy groups, in section $\mathrm{V}$ we develop the technique of spectral sequences, in order to recover differential forms on the bundle from forms on the fiber. Besides, the spectral sequences will aid us in giving a meaning to the notion of "adaptation to the connection."

Section VI finally summarizes our results especially with regard to the description of the monopole-induced proton decay, and indicates generalizations to non-abelian Yang-Mills theories.

\section{THE UNGAUGED SKYRME MODEL}

The Skyrme model ${ }^{1}$ in theoretical nuclear physics is a chiral invariant effective field theory modelled to describe the low energy limit of QCD. Let $N_{F}$ denote the number of flavors in QCD (we will use Einstein summation convention, where greek indices label space-time coordinates running from 0 to 3 , whereas latin indices start from 1, e.g. for space coordinates). Then defined by

$$
U=\exp \left(i \pi^{a} \lambda_{a}\right) \quad \text { with } \quad \lambda_{a}=\left(\lambda_{a}\right)^{\dagger} \in \mathrm{C}^{N_{F} \times N_{F}}, \operatorname{Tr}\left(\lambda_{a}\right)=0,
$$

the meson fields $\pi^{a}$ generate differentiable functions $U: M \rightarrow \mathrm{SU}_{N_{F}}$ from space-time $M$ to the group of unitary $N_{F} \times N_{F}$-matrices of determinant 1 . The vacuum is represented by the unit matrix $1 \in \mathrm{SU}_{N_{F}}$. Requiring $\pi^{a}(r) \rightarrow 0$ and thus $U(r) \rightarrow 1$ for $r \rightarrow \infty$ one can compactify euclidian space $\mathbf{R}^{3}$, resp., space-time $\mathbf{R}^{4}$, so that the meson fields constitute functions

$$
U: \mathbb{R}_{(t)} \times \mathrm{S}^{3} \rightarrow \mathrm{SU}_{N_{F}}, \quad \text { resp., } \quad U: \mathrm{S}^{4} \rightarrow \mathrm{SU}_{N_{F}}
$$

From this it is obvious that the topology of the unitary groups plays a crucial role in all questions concerning the topological properties of the Skyrme model. We will therefore collect some results on the homotopy groups and the differential forms on $\mathrm{SU}_{m}$, resp., $\mathrm{U}_{m}$. About the (stable) homotopy groups $\pi_{n}$ it is known from Bott's periodicity theorem ${ }^{4}$ that for all $m>n \in \mathrm{N}$

$$
\begin{gathered}
\pi_{2 n}\left(\mathrm{SU}_{m}\right)=\pi_{2 n}\left(\mathrm{U}_{m}\right)=0, \\
\pi_{2 n+1}\left(\mathrm{SU}_{m}\right) \cong \pi_{2 n+1}\left(\mathrm{U}_{m}\right) \cong \mathbb{Z} .
\end{gathered}
$$

Reference 9 exhibits explicit representatives for the elements of $\pi_{2 n+1}\left(\mathrm{SU}_{m}\right), m>n<3$.

As for the differential forms, we will give an introduction into what physicists call the "current algebra approach;" we shall need certain basic results which, for the benefit of the reader, we will also survey in the following subsection.

\section{A. Differential forms on the unitary groups}

Let $\mathrm{U}_{m}$ be embedded into $\mathrm{Gl}\left(\mathrm{C}^{m}\right)$ by $i: \mathrm{U}_{m} \rightarrow \mathrm{Gl}\left(\mathrm{C}^{m}\right)$. With $L:=U^{-1} d U=U^{\dagger} d U$ and $R:=(d U) U^{-1}=(d U) U^{\dagger} \in \mathscr{A}_{1}\left(\mathrm{U}_{m}, \mathrm{C}^{m \times m}\right)$ we denote the left, resp., right invariant currents: $L$ and $R$ are the pullbacks $i^{\star} \Theta_{\mathrm{Gl}\left(\mathrm{C}^{m}\right)}^{L}$, resp., $i^{\star} \Theta_{\mathrm{Gl}\left(\mathrm{C}^{m}\right)}^{R}$, where for any Lie group $G$ with Lie algebra $\mathfrak{g}=\mathrm{L}(G), \Theta_{G}^{L}$ and $\Theta_{G}^{R} \in \mathscr{l}_{1}(G, \mathfrak{g})$ mean the left, resp., right canonical $\mathfrak{g}$-valued 1-forms on $G$. Thus $L$ and $R$ are $\mathrm{C}^{m \times m}$-valued 1 -forms that are invariant under multiplication with constant 
elements of $U_{m}$ from the left, resp., from the right and obey $L(X)(1)=R(X)(1)=X_{1}$ for all vector fields $X \in \mathscr{D}^{1}\left(\mathrm{U}_{m}\right)$ with $X(\mathrm{l})=X_{1} \in \mathfrak{u}_{m}=\mathrm{L}\left(\mathrm{U}_{m}\right)=T_{1}\left(\mathrm{U}_{m}\right)$. For any constant $Q \in \mathrm{C}^{m \times m}$, we define $\lambda_{k}^{Q}$ and $\rho_{k}^{Q} \in \mathscr{A}_{k}\left(\mathrm{U}_{m}, \mathrm{C}\right)$ by

$$
\begin{aligned}
& \lambda_{k}^{Q}:=\operatorname{Tr}\left(Q L^{k}\right):=\operatorname{Tr} \underbrace{(Q L \wedge \cdots \wedge L)}_{k}, \\
& \rho_{k}^{Q}:=\operatorname{Tr}\left(Q R^{k}\right):=\operatorname{Tr} \underbrace{(Q R \wedge \cdots \wedge R)}_{k},
\end{aligned}
$$

where $\wedge$ is the wedge product of differential forms. These are left, resp., right invariant complexvalued $k$-forms on $\mathrm{U}_{m}$; for $Q=1$ we have

$$
\omega_{k}:=\lambda_{k}^{1}=\rho_{k}^{1}=\operatorname{Tr}\left(L^{k}\right)=\operatorname{Tr}\left(R^{k}\right) \in \mathscr{f}_{k}\left(\mathrm{U}_{m}, \mathrm{C}\right),
$$

which is invariant under all "chiral" $\left(\mathrm{SU}_{m}\right)_{L} \times\left(\mathrm{SU}_{m}\right)_{R}$ transformations. For any differentiable $U: M \rightarrow \mathrm{U}_{m}$ we get the pullbacks (here $L_{i}=U^{\dagger} \partial_{i} U$ )

$$
\begin{gathered}
U^{\star} \lambda_{k}^{Q}=\operatorname{Tr}\left(Q L_{i_{1}} \cdots L_{i_{k}}\right) d x^{i_{1}} \wedge \cdots \wedge d x^{i_{k}} \in \mathscr{B}_{k}(M, \mathrm{C}), \\
U^{\star} \rho_{k}^{Q}=\operatorname{Tr}\left(Q R_{i_{1}} \cdots R_{i_{k}}\right) d x^{i_{1}} \wedge \cdots \wedge d x^{i_{k}} \in \mathscr{B}_{k}(M, \mathrm{C}), \\
U^{\star} \omega_{k}=\operatorname{Tr}\left(L_{i_{1}} \cdots L_{i_{k}}\right) d x^{i_{1}} \wedge \cdots \wedge d x^{i_{k}} \in \mathscr{B}_{k}(M, \mathrm{C})
\end{gathered}
$$

(pullbacks commute with $d$ and $\wedge$ ). Because of the symmetry of the trace and the antisymmetry of the wedge product, $\omega_{k}=0$ for even $k=2 l$, and so is $U^{\star} \omega_{k}=0 . L$ and $R$ obey the Maurer-Cartan identities, that read $d L=-L \wedge L, d R=+R \wedge R$ or, equivalently,

$$
\begin{aligned}
& d\left(U^{\star} L\right)=-\left(U^{\star} L\right) \wedge\left(U^{\star} L\right)=-L_{i_{1}} L_{i_{2}} d x^{i_{1}} \wedge d x^{i_{2}}=-\frac{1}{2}\left[L_{i_{1}}, L_{i_{2}}\right] d x^{i_{1}} \wedge d x^{i_{2}} \\
& d\left(U^{\star} R\right)=+\left(U^{\star} R\right) \wedge\left(U^{\star} R\right)=+R_{i_{1}} R_{i_{2}} d x^{i_{1}} \wedge d x^{i_{2}}=+\frac{1}{2}\left[R_{i_{1}}, R_{i_{2}}\right] d x^{i_{1}} \wedge d x^{i_{2}}
\end{aligned}
$$

Illustrating that the exterior differentiation $d$ is a differential operator $\left(d^{2}=0\right)$, this yields

$$
\begin{gathered}
d L^{2 l+1}=-L^{2 l+2}, \quad d R^{2 l+1}=R^{2 l+2}, \quad d L^{2 l+2}=d R^{2 l+2}=0, \\
d\left(U L^{2 l}\right)=U L^{2 l+1}, \quad d\left(L^{2 l} U^{\dagger}\right)=-L^{2 l+1} U^{\dagger}, \quad d\left(U L^{2 l+1}\right)=d\left(L^{2 l+1} U^{\dagger}\right)=0,
\end{gathered}
$$

for $l \in \mathbf{N}_{0}$, and we thus have the following lemma:

Lemma II.1: If $\alpha_{r} \in \mathcal{A}_{r}\left(\mathrm{U}_{m}, \mathrm{C}\right)$ is any $r$-form on $\mathrm{U}_{m}$, then

$$
\begin{aligned}
& d\left(\alpha_{r} \wedge \lambda_{k}^{Q}\right)=d \alpha_{r} \wedge \lambda_{k}^{Q}+ \begin{cases}(-1)^{r+1} \alpha_{r} \wedge \lambda_{k+1}^{Q} \in \mathscr{A}_{r+k+1}\left(\mathrm{U}_{m}, \mathrm{C}\right), & k \text { odd }, \\
0, & k \text { even, }\end{cases} \\
& d\left(\alpha_{r} \wedge \rho_{k}^{Q}\right)=d \alpha_{r} \wedge \rho_{k}^{Q}+ \begin{cases}(-1)^{r} \alpha_{r} \wedge \rho_{k+1}^{Q} \in \mathscr{A}_{r+k+1}\left(\mathrm{U}_{m}, \mathrm{C}\right), & k \text { odd }, \\
0, & k \text { even. }\end{cases}
\end{aligned}
$$

Equivalently, if $U: M \rightarrow \mathrm{U}_{m}$ is differentiable and $\beta_{r} \in \mathscr{A}_{r}(M, \mathrm{C})$,

$$
d\left(\beta_{r} \wedge U^{\star} \lambda_{k}^{Q}\right)=d \beta_{r} \wedge U^{\star} \lambda_{k}^{Q}+\left\{\begin{array}{l}
(-1)^{r+1} \beta_{r} \wedge U^{\star} \lambda_{k+1}^{Q} \in \mathcal{A}_{r+k+1}(M, C), \quad k \text { odd }, \\
0, \quad k \text { even, }
\end{array}\right.
$$




$$
d\left(\beta_{r} \wedge U^{\star} \rho_{k}^{Q}\right)=d \beta_{r} \wedge U^{\star} \rho_{k}^{Q}+\left\{\begin{array}{l}
(-1)^{r} \beta_{r} \wedge U^{\star} \rho_{k+1}^{Q} \in \mathscr{A}_{r+k+1}(M, \mathrm{C}) \quad k \text { odd }, \\
0, \quad k \text { even. }
\end{array}\right.
$$

Corollary 1I.2: The forms $\omega_{k}$ are closed: $d \omega_{k}-0$, resp., $d\left(U^{\star} \omega_{k}\right)-0$.

Moreover, one can show ${ }^{5}$ that there is no differential form $\alpha_{2 n} \in \mathscr{C}_{2 n}\left(\mathrm{U}_{m}, \mathrm{C}\right)$ with $\omega_{2 n+1}=d \alpha_{2 n}$, i.e. the $\omega_{2 n+1}$ are not exact, and that they generate the de-Rham cohomology of the closed forms, modulo the exact forms on $\mathrm{SU}_{m}$, resp., $\mathrm{U}_{m}$, which is homeomorphic to $\mathrm{S}^{\mathrm{l} \times \mathrm{SU}} \mathrm{U}_{m}$ :

Theorem II.3: The de-Rham cohomology of the unitary groups

$$
H^{*}\left(\mathrm{U}_{m}, \mathrm{C}\right) \cong H^{*}\left(\mathrm{~S}^{1}, \mathrm{C}\right) \otimes H^{*}\left(\mathrm{SU}_{m}, \mathrm{C}\right),
$$

resp., $H^{*}\left(\mathrm{SU}_{m}, \mathrm{C}\right)$ is generated by $\omega_{1}, \omega_{3}, \ldots, \omega_{2 m-1}$, resp., $i^{\star} \omega_{3}, \ldots, i^{\star} \omega_{2 m-1}$, with inclusion $i: \mathrm{SU}_{m} \rightarrow \mathrm{U}_{m}$.

\section{B. Baryons in the Skyrme model}

Baryons appear as topological soliton solutions-as "skyrmions"-of the meson fields. The number $B$ of baryons described by a given mesonic field configuration $U$ can be computed by an integration over the space manifold:

$$
B(U)=\int_{S^{3}}-\frac{1}{24 \pi^{2}} U^{\star} \omega_{3}=\int_{\mathbf{R}^{3}}-\frac{1}{24 \pi^{2}} \operatorname{Tr}\left(L_{i} L_{j} L_{k}\right) d x^{i} \wedge d x^{j} \wedge d x^{k} .
$$

Compactification of space-time is crucial for the existence of nontrivial soliton solutions. Normally there is no guarantee that the integral in (5) is an integer, but for spheres we have the following index theorem. ${ }^{10}$ Let $\epsilon^{i_{1} i_{2} \cdots i_{k}}$ denote the totally antisymmetrical Levi-Civita symbol, then we have

Theorem II.4: For every map $U: \mathrm{S}^{2 n-1} \rightarrow \mathrm{U}_{m}$ the integral

$$
\begin{aligned}
& n(U)=\int_{\mathrm{S}^{2 n-1}}\left(\frac{i}{2 \pi}\right)^{n} \frac{(n-1) !}{(2 n-1) !} U^{\star} \omega_{2 n-1}
\end{aligned}
$$

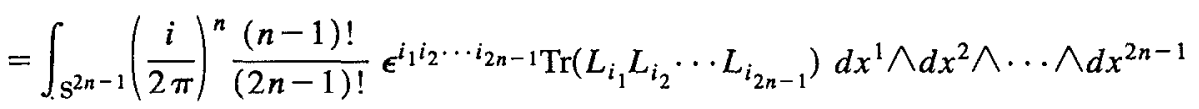

is an integer. The assignment $[U] \mapsto n(U): \pi_{2 n-1}\left(\mathrm{U}_{m}\right) \rightarrow \mathrm{Z}$ is an isomorphism for $m \geqslant n$.

We are thus able to identify $(i / 2 \pi)^{n}[(n-1) ! /(2 n-1) !] \omega_{2 n-1}$ with the generators of

$$
H^{*}\left(\mathrm{U}_{m}, \mathrm{Z}\right) \cong H^{*}\left(\prod_{l=0}^{m-1} \mathrm{~S}^{2 l+1}, \mathrm{Z}\right), \text { resp., } H^{*}\left(\mathrm{SU}_{m}, \mathrm{Z}\right) \cong H^{*}\left(\prod_{l=1}^{m-1} \mathrm{~S}^{2 l+1}, \mathrm{Z}\right)
$$

At any time $t$ the meson fields form differentiable functions $U(t, \cdot): \mathrm{S}^{3} \rightarrow \mathrm{SU}_{N_{F}}$ and thus represent elements of the homotopy groups $\pi_{3}\left(\mathrm{U}_{N_{F}}\right) \cong \mathrm{Z}$ for $N_{F} \geqslant 2$. Although these fields need not be constant in time, continuity forces them to change only within their equivalence class of homotopic functions. Thus the integer characterizing the homotopy class is a topological invariant, the "topological charge," that can be interpreted as the number of baryons and be computed by (5), as Theorem II.4 tells us.

The vacuum map represents the zero element, and so $B(U \equiv 1)=0$. For proton and neutron we have $B=1$, for their antiparticles $B=-1$. Annihilation of proton and antiproton corresponds to the "addition" of their maps within the homotopy group and generates a mesonic field of topological charge $B=0$. 


\section{The action integral}

The meson fields obey the field equations derived as Euler-Lagrange equations from a lagrangian $\mathscr{L}(U, d U)$ by variation of the action integral $\Gamma(U)=\int_{S^{4}} \mathscr{L} d V$. The latter splits into two parts (we set $\hbar=1$ ): the nonanomalous action $\Gamma_{N A}(U)$

$$
=\int_{S^{4}}\left(-\frac{f_{\pi}^{2}}{4} \operatorname{Tr}\left(L_{\mu} L^{\mu}\right)+\frac{1}{32 a^{2}} \operatorname{Tr}\left(\left[L_{\mu}, L_{\nu}\right]\left[L^{\mu}, L^{\nu}\right]\right)\right) d V
$$

where $f_{\pi}$ is the pion decay constant and $a^{-2}$ a coupling constant, and the Wess-Zumino term ${ }^{11}$ $\left(N_{C}\right.$ is the number of colors in QCD)

$$
\Gamma_{W Z}(U)=\frac{i N_{C}}{240 \pi^{2}} \int_{D^{5}}\left(U^{\prime}\right)^{\star} \omega_{5}
$$

that describes the anomalous processes of QCD. Now $H^{5}\left(\mathrm{SU}_{2}, \mathrm{C}\right)=0$, so the Wess-Zumino term only contributes to the total action for $N_{F} \geqslant 3$. In that case one uses $\pi_{4}\left(\mathrm{SU}_{N_{F}}\right)=0$ and extends $U$ to a differentiable map $U^{\prime}: D^{5} \rightarrow \mathrm{SU}_{3}$ from a five-dimensional disk $D^{5}$ whose boundary $\partial D^{5}$ is space-time $S^{4}$. The topological quantization of the coupling constant $\lambda=i N_{C} / 240 \pi^{2}$ is again a consequence of Theorem II.4, and of the requirement that for any extension $U^{\prime}$ the result has to be unique. This forces [note $\pi_{5}\left(\mathrm{SU}_{N_{F}}\right) \cong \mathrm{Z}$ for $N_{F} \geqslant 3$ ]

$$
\lambda \int_{\mathrm{S}^{5}}(\tilde{U})^{\star} \omega_{5}=2 \pi z, \quad z \in \mathbb{Z}
$$

where $\mathrm{S}^{5}$ is the 5-sphere which one obtains by glueing any two 5-cells $D_{(1)}^{5}$ and $D_{(2)}^{5}$ at space-time $\mathrm{S}^{4}=\partial D_{(1)}^{5}=\partial D_{(2)}^{5}$ together, and where we have defined $\tilde{U}=U_{(1)}^{\prime} \cup U_{(2)}^{\prime}: \mathrm{S}^{5} \rightarrow \mathrm{SU}_{3}$ as the corresponding extension to this 5 -sphere. ${ }^{6}$

The so-called hedgehog ansal ${ }^{12}$ leads to a numerical solution of the field equations for all $B \in \mathrm{Z}$. The action is invariant under all chiral transformations $U \mapsto g_{L} U g_{R}^{-1}$ with $g_{L}, g_{R} \in \mathrm{SU}_{N_{F}}$. This symmetry is spontaneously broken: the vacuum state is only invariant under diagonal $\mathrm{SU}_{N_{F}}$ transformations $U \mapsto V U V^{-1}$. One can add further chiral invariant terms of fourth order to the nonanomalous lagrangian

$$
\frac{1}{32 f^{2}} \operatorname{Tr}\left(\left\{L_{\mu}, L_{\nu}\right\}\left\{L^{\mu}, L^{\nu}\right\}\right)+\frac{1}{32 g^{2}} \operatorname{Tr}\left(\partial_{\mu} L_{\nu} \partial^{\mu} L^{\nu}\right),
$$

with coupling constants $f^{2}$ and $g^{2}$ and anticommutator braces $\{$,$\} , or-in order to take the finite$ pion mass $M_{\pi}$ into account-a mass term, breaking the axial symmetry $\left(f_{\pi}^{2} M_{\pi}^{2} / 2\right) \operatorname{Tr}(U-1)$, resp.,

$$
\frac{f_{\pi}^{2} M_{\pi}^{2}}{2\left(m_{u}+m_{d}\right)} \operatorname{Tr}\left(M_{q}\left(U+U^{\dagger}-2 \cdot 1\right)\right)
$$

for $N_{F}=2$, resp., 3, where $M_{q}=\operatorname{diag}\left(m_{u}, m_{d}, m_{s}\right)$ is the quark mass matrix, and $m_{u}, m_{d}, m_{s}$ denote the masses of up, down, and strange quarks, resp.

\section{Conservation of the baryonic current}

$d \omega_{3}=0$ allows for the conservation of the baryonic current within the Skyrme model. On every $n$-dimensional pseudo-Riemannian oriented manifold $M$ [i.e. equipped with a volume form $\left.d V \in \mathscr{A}_{n}(M)\right]$ the Hodge star operator $*: \not \mathscr{A}(M) \rightarrow \mathcal{A}(M), \mathscr{f}_{p}(M) \rightarrow \mathscr{A}_{n-p}(M)$ is uniquely defined by ${ }^{5,13,14}$ 


$$
\beta_{p} \mapsto * \beta_{p}: \alpha_{p} \wedge * \beta_{p}=\left\langle\alpha_{p}, \beta_{p}\right\rangle d V \text { for all } \alpha_{p} \in \mathscr{C}_{p}(M)
$$

where $\langle\cdot, \cdot\rangle$ is the scalar product of $p$-forms induced by the metric $\tilde{g}$ on $M . *$ serves to establish the co-derivation $\delta: A_{A}(M) \rightarrow . A(M), \mathscr{A}_{p}(M) \rightarrow A_{p-1}(M)$ and the Laplace-Beltrami operator $\Delta: A(M) \rightarrow \mathscr{A}(M), \mathscr{A}_{p}(M) \rightarrow \mathscr{A}_{p}(M)$ defined by

$$
\begin{aligned}
& \delta \omega_{p}:=-(-1)^{n(p-1)} \operatorname{sgn}(\tilde{g}) * d * \omega_{p}, \\
& \Delta \omega_{p}:-d \delta \omega_{p}+\delta d \omega_{p}=(\delta+d)^{2} \omega_{p} .
\end{aligned}
$$

Like $d, \delta$ is a differential operator: $\delta^{2}=0$. The local expressions might be more familiar. On a chart $U_{\alpha} \subset M$ with coordinates $x^{1}, \ldots, x^{n}$ and local evaluations $\left.\omega_{p}\right|_{U_{\alpha}}=:(1 / p !) \omega_{i_{1}} \cdots i_{p} d x^{i_{1}}$ $\wedge \ldots \wedge d x^{i_{p}},\left.\tilde{g}\right|_{U_{\alpha}}=: g_{i j} d x^{i} d x^{j}$ with maps $\omega_{i_{1}} \ldots i_{p}, g_{i j} \in C^{\infty}\left(U_{\alpha}\right)$ and $g:=\operatorname{det}\left(g_{i j}\right)$ we have

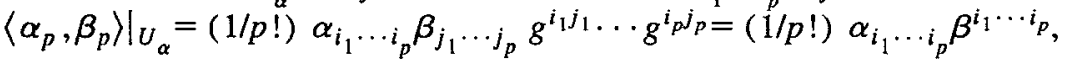

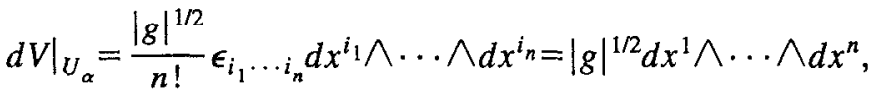

$$
\begin{aligned}
& \left.d \omega_{p}\right|_{U_{\alpha}}=\frac{1}{p !} \partial_{i_{0}} \omega_{i_{1}} \ldots i_{p} d x^{i_{0}} \wedge d x^{i_{1}} \wedge \cdots \wedge d x^{i_{p}} \\
& \left.* \omega_{p}\right|_{U_{\alpha}}=\frac{|g|^{1 / 2}}{(n-p) !}\left(\frac{1}{p !} \omega^{i_{1} \cdots i_{p}} \epsilon_{i_{1}} \ldots i_{n}\right) d x^{i_{p+1}} \wedge \ldots \wedge d x^{i_{n}}, \\
& \left.\delta \omega_{p}\right|_{U_{\alpha}}=\frac{(-1)^{p}}{(p-1) !}|g|^{-1 / 2} \partial_{j_{p}}\left(|g|^{1 / 2} \omega^{j_{1}} \cdots j_{p}\right) \cdot g_{i_{1} j_{1}} \cdots g_{i_{p-1} j_{p-1}} d x^{i_{1}} \wedge \ldots \wedge d x^{i_{p-1}} \\
& =\frac{(-1)^{p}}{(p-1) !} \partial^{i_{p}}\left(\omega_{i_{1} \cdots i_{p}}\right) d z^{i_{1}} \wedge \ldots \wedge d z^{i_{p-1}} \\
& \left.\Delta \omega_{p}\right|_{U_{\alpha}}=-\frac{1}{p !} \partial^{j} \partial_{j} \omega_{i_{1} \cdots i_{p}} d z^{i_{1}} \wedge \ldots \wedge d z^{i_{p}}
\end{aligned}
$$

where the last two equalities hold for cartesian coordinates $z^{i}$ only.

Now the baryonic current on space-time $M$ is defined by $\tilde{B}:=*\left(\left(-1 / 4 \pi^{2}\right) U^{\star} \omega_{3}\right) \in \mathscr{A}_{1}(M)$. Locally this means $\tilde{B}=B_{\mu} d x^{\mu}=\left(1 / 24 \pi^{2}\right) \epsilon_{\mu}{ }^{\nu \rho \sigma}|g|^{1 / 2}$ $\times \operatorname{Tr}\left(L_{\nu} L_{\rho} L_{\sigma}\right) d x^{\mu}$, where on space-time with signature $(+---)$ we have $\epsilon^{0123}=\epsilon_{0}{ }^{123}=-|g|^{-1} \epsilon_{0123}=-|g|^{-1}$. This yields $B(U)=\int_{M_{(x)}} B^{0} d V$ where $M_{(\vec{x})}$ denotes the space manifold and $\tilde{B}$ is co-closed by Corollary II.2.:

$$
\delta \tilde{B}=\frac{-1}{4 \pi^{2}} * d * *\left(U^{\star} \omega_{3}\right)=\frac{-1}{4 \pi^{2}} * d\left(U^{\star} \omega_{3}\right)=0
$$

Locally this is exactly the conservation law for the baryonic current $\partial_{\mu}\left(|g|^{1 / 2} B^{\mu}\right)=0$ or, in cartesian coordinates, $\partial_{\mu} B^{\mu}=0$, where $B^{\mu}=\left(1 / 24 \pi^{2}\right) \epsilon^{\mu \nu \rho \sigma} \operatorname{Tr}\left(L_{\nu} L_{\rho} L_{\sigma}\right)$. Using Stokes's theorem one again verifies the conservation of the number of baryons

$$
\frac{\partial}{\partial t} B(U)=\int_{M_{(\vec{x})}} \partial_{0} B^{0} d V=\int_{M_{(\vec{x})}} \partial_{i}\left(|g|^{1 / 2} B^{i}\right) d x^{1} \wedge d x^{2} \wedge d x^{3}=\int_{\partial M_{(\vec{x})}} B^{i} d A^{i}=0
$$

whenever $\partial M_{(\vec{x})}=0$ or the baryonic current vanishes there. 
In contrast to Noether currents this conservation law is independent of the validity of the field equations but a mere consequence of the topology of the underlying manifolds (e.g. compactification of $M_{(\vec{x})}$.

\section{MONOPOLES AND FIBER BUNDLES}

\section{A. Maxwell's equations in differential geometric form}

It is well known that Maxwell's equations (in their matter-free form) can conveniently be presented by use of differential forms. ${ }^{5,14,15}$ If we define

$$
\begin{aligned}
& \text { electric 1-form } E,\left.\quad E\right|_{U}=E_{\mu} d x^{\mu}, \quad E_{\mu}:=(0, \vec{E}), \\
& \text { magnetic 1-form } B,\left.\quad B\right|_{U}=B_{\mu} d x^{\mu}, \quad B_{\mu}:=(0, \vec{B}), \\
& \text { source 1-form } J,\left.\quad J\right|_{U}=J_{\mu} d x^{\mu}, \quad J_{\mu}:=(-c \rho, \vec{j}), \\
& \text { gauge potential 1-form } A,\left.\quad A\right|_{U}=A_{\mu} d x^{\mu}, \quad A_{\mu}:=(-\Phi, \vec{A}) \text {, } \\
& \text { Faraday 2-form } F,\left.\quad F\right|_{U}=\frac{1}{2} F_{\mu \nu} d x^{\mu} \wedge d x^{\nu},
\end{aligned}
$$

where $F=(c / 2)[(E \wedge d t-d t \wedge E)+*(B \wedge d t-d t \wedge B)]$, Maxwell's equations

$$
\begin{aligned}
\vec{\nabla} \times \vec{E}+\frac{1}{c} \frac{\partial}{\partial t} \vec{B} & =0, \quad \vec{\nabla} \times \vec{B}-\frac{1}{c} \frac{\partial}{\partial t} \vec{E}=\frac{4 \pi}{c} \vec{j}, \\
\vec{\nabla} \cdot \vec{B} & =0, \quad \vec{\nabla} \cdot \vec{E}=4 \pi \rho,
\end{aligned}
$$

simply read $d F=0, \delta F=-(4 \pi / c) J$. The continuity equation $\vec{\nabla} \cdot \vec{j}+(\partial / \partial t) \rho=0$ reads $\delta J=0$ and is a consequence of $\delta^{2}=0$. Introducing gauge potentials by $\vec{B}=\vec{\nabla} \times \vec{A}, \vec{E}=-\vec{\nabla} \Phi-(1 / c)$ $\times(\partial / \partial t) \vec{A}$ is equivalent to $F=d A$, from which $d F=0$ then follows by $d^{2}=0$. Finally the Lorentz gauging $\vec{\nabla} \cdot \vec{A}+(1 / c)(\partial / \partial t) \Phi=0$ reads $\delta A=0$, yielding $\Delta A=-(4 \pi / c) J$. Wherever $J=0$, the Faraday 2-form is harmonic: $\Delta F=0$, which is equivalent to the wave equation, and under Lorentz gauging $A$ is harmonic, too.

Lorentz transformations $L: M \rightarrow M$ are isometries of $M$, i.e. $L^{\star} \tilde{g}=\tilde{g}$ and thus not only $d$ but also $\delta$ and $\Delta$ commute with $L$. This proves Lorentz invariance of all these equations connecting $F, A$ and $J$, where $F^{\prime}=L^{\star} F, A^{\prime}=L^{\star} A$ and $J^{\prime}=L^{\star} J$ are the quantities in the new system.

Yet, this is not sufficient for the description of magnetic monopoles. Given any closed $F$ $\in \mathscr{H}_{2}(M)$ the existence of a gauge form $A$ satisfying $d A=F$ depends on the topology of spacetime: it is guaranteed only for $H^{2}(M, \mathbb{R})=0$. If we exclude single points out of the space manifold then there exist (global) electromagnetic fields-namely those of magnetic (anti-)monopoles located in these points-for which no global gauge potentials exist.

We are interested in the case of a single (anti-) monopole of magnetic charge $m \cdot g_{D}$ (at first let $m \in \mathbb{R})$, which rests in the origin of our space manifold. Then $M \cong \mathbb{R}_{(t)} \times \mathbb{R}_{(r)}^{+} \times S_{(\theta, \phi)}^{2}$ and $H^{2}(M, \mathbb{R}) \cong H^{2}\left(\mathrm{~S}^{2}, \mathbb{R}\right) \cong \mathbf{R}$. The resulting 2 -form related to $\vec{E}=0$ and $\vec{B}=m\left(g_{D} / r^{2}\right) \overrightarrow{e_{r}}$ is

$$
F_{M}=m g_{D} \sin \theta d \theta \wedge d \phi=2 m g_{D} \sin \frac{\theta}{2} \cos \frac{\theta}{2} d \theta \wedge d \phi .
$$

Gauge forms exist only locally on the northern, resp., southern hemisphere:

$$
A_{\mathrm{M}}^{(+)}=2 m g_{D} \sin ^{2} \frac{\theta}{2} d \phi \text { and } A_{\mathrm{M}}^{(-)}=-2 m g_{D} \cos ^{2} \frac{\theta}{2} d \phi,
$$


where $A_{M}^{(+)}$becomes singular at the south pole $(\theta=\pi)$ and vice versa.

Yet it is possible to define a global 1-form that contains the gauge potentials, but this is no form on the space-time manifold. In order to do this and to describe interactions between monopoles and baryons it is profitable to use fiber bundles.

\section{B. Fiber bundles}

Fiber bundles $B(M, F, G)$ are generalizations of the direct product of two spaces (we only consider $C^{\infty}$-manifolds): $B$ is the bundle manifold, $M$ the base, $F$ the fiber and $G$ the group of the bundle. ${ }^{13,15,16}$ Only one global projection $\pi: B \rightarrow M$ exists. We have a bundle atlas $\left\{\left(U_{\alpha}, \psi_{\alpha}\right)\right\}_{\alpha \in A}$ consisting of bundle charts $\left(U_{\alpha}, \psi_{\alpha}\right)$, where $\mathfrak{U}=\left\{U_{\alpha}\right\}_{\alpha \in A}$ is an open cover of $M$ and $\psi_{\alpha}: \pi^{-1}\left(U_{\alpha}\right) \rightarrow U_{\alpha} \times F: b \mapsto\left(\pi(b), \pi_{\alpha}(b)\right)$ are local trivializations with local projections $\pi_{\alpha}: \pi^{-1}\left(U_{\alpha}\right) \rightarrow F$ on the fiber. Via these diffeomorphisms $\psi_{\alpha}$ we will frequently identify $\pi^{-1}\left(U_{\alpha}\right)$ and $U_{\alpha} \times F$. If a global $\left(C^{\infty}\right)$-projection on the fiber exists then $B \cong M \times F$ and the bundle is trivial.

$G$ is an effective topological transformation group on $F$, acting on $F$ from the left: $g \triangleleft(h \triangleleft f)=(g \cdot h) \triangleleft f \forall g, h \in G, \forall f \in F$. For all $x \in U_{\alpha}$ let $\pi_{\alpha, x}:=\left.\pi_{\alpha}\right|_{\pi^{-1}(\{x\})}$. Then for all $\alpha, \beta \in A$ and all $x \in U_{\alpha} \cap U_{\beta}$ the diffeomorphism $\pi_{\alpha, x}{ }^{\circ} \pi_{\beta, x}^{-1}: F \rightarrow F$ defines an element $g_{\alpha \beta}(x)$ $\in G$, and we thus have $C^{\infty}$-maps $g_{\alpha \beta}: U_{\alpha} \cap U_{\beta} \rightarrow G$. $\left[g, g_{\alpha \beta}\right.$ and $g_{\alpha \beta}(x)$ are not to be mixed up with the metric tensor, there will be no summation over $\alpha$ and $\beta$.]

For abelian $G$ the left action on $F$ defines a left action on the whole bundle:

$$
g \triangleleft b:=L_{g}(b):=\psi_{\alpha}^{-1}\left(\pi(b), g \triangleleft \pi_{\alpha}(b)\right)
$$

for all $g \in G$ and $b \in B$, where $\pi(b) \in U_{\alpha}$, is then well defined and fiber preserving: $\pi(g \triangleleft b)=\pi(b)$.

Note III.1: The maps $g_{\alpha \beta}$ are crucial for the global structure of the bundle. If the base $M$ with an open cover $\mathfrak{U}=\left\{U_{\alpha}\right\}_{\alpha \in A}$ and the fiber $F$ are given then the $g_{\alpha \beta}$ define the whole bundle up to equivalences. ${ }^{15,16}$

Examples for bundles are the Moebius strip and the tangent bundle of a manifold $T(M)$ consisting of all tangent vectors at all points in $M$, where $M$ is the $n$-dimensional base manifold, $\mathbf{R}^{n}$ is the fiber and $\operatorname{Gl}\left(\mathbf{R}^{n}\right)$ is the group of the bundle. Analogously we have the cotangent bundle $T^{*}(M)$, that consists of all cotangent vectors at all points in $M$.

Sections are $C^{\infty}$-maps $\sigma: M \rightarrow B: x \mapsto \sigma(x) \in \pi^{-1}(x)$. Normally only local sections exist: fixing $y \in F$ we have $\sigma_{\alpha, y}: U_{\alpha} \rightarrow \pi^{-1}\left(U_{\alpha}\right): x \mapsto \psi_{\alpha}^{-1}(x, y)$, but for tangent, resp., cotangent bundles global sections always exist, these are exactly the vector fields, resp. the 1 -forms on $M$.

By a principal fiber bundle $P(M, G)$ we mean a bundle where $G=F$ acting on itself by left multiplication. In addition we have a free fiber preserving right action on $P$ defined by

$$
p \triangleright g:=R_{g}(p):=\psi_{\alpha}^{-1}\left(\pi(p), \pi_{\alpha}(p) \cdot g\right)
$$

for all $g \in G$ and $p \in P$, where $\pi(p) \in U_{\alpha}$, which is independent of the choice of $\alpha$ because left and right multiplication on $G$ commute.

Given any bundle $B(M, F, G)$ one can construct an associated principal bundle $P$ by taking $M=\cup_{\alpha \in A} U_{\alpha}, G$ and the maps $g_{\alpha \beta}$ but choosing $G$ as fiber (Note III.1). On the product manifold $P \times F$ let $G$ act on the right: $\tilde{R}_{g}(p, f):=\left(p \triangleright g, g^{-1} \triangleleft f\right) ; P \times{ }_{G} F$ denotes the quoticnt space of $P \times F$ by this action. Then $B \cong P \times_{G} F{ }^{17}$ Two bundles are called associated if their associated principal bundles coincide.

Principal bundles are trivial iff global sections exist. In order to decide whether a given bundle is trivial or not one can construct the associated principal bundle and look for sections there.

The physics of the skyrmion bundle, and of any other theory that involves fiber bundles and differential geometry on them, e.g. Yang-Mills theories, can only be understood within the frame- 
work of connections on principal and associated bundles. Since these topics are not available in the physical literature in full detail, and for notational convenience, the following two subsections present their basics and applications in view of our purposes. The reader familiar with differential geometry on fiber bundles may skip these subsections.

\section{Differential geometry on principal bundles}

Every bundle chart for a fiber bundle $B$ also induces a local trivialization of the tangent bundle of the given bundle: every tangent space splits into the direct product of the horizontal and the vertical subspace: $T_{b}(B) \cong H_{b} \oplus V_{b}$. Only the latter, consisting of all vectors tangential to the fiber, is given naturally and thus globally: if $\pi_{\star}$ (the push-out of $\pi$ ) denotes the map between the tangent spaces induced by $\pi$ (also called the differential $d \pi$ ), then

$$
V_{b}=\operatorname{ker}\left(\pi_{\star}\right)_{b} \text { with }\left(\pi_{\star}\right)_{b}: T_{b}(B) \rightarrow T_{\pi(b)}(M) \forall b \in B .
$$

We thus have the vertical bundle $V(B)$ as subbundle of $T(B) ; v \mathscr{V}^{\mathrm{1}}(B)$ denotes the set of all its sections, i.e. the vertical vector fields.

On principal bundles let $\tau_{p}: G \rightarrow P: g \mapsto \tau_{p}(g):=p \triangleright g$ denote the $C^{\infty}$-map given by (12). Then $d \tau_{p}:=\left(\left(\tau_{p}\right)_{\star}\right)_{e}: \mathfrak{g} \rightarrow T_{p}(P): A \mapsto d \tau_{p}(A)=A^{*}(p)$ ( $e$ is the neutral element in $G$ ) is a linear isomorphism between $\mathfrak{g}=\mathrm{L}(G)$ and $V_{p}$, and if $A^{*} \in \cup \mathscr{D}^{1}(P)$ denotes the induced vector field on $P$ we have $\left(R_{g}\right)_{\star} A^{*}=\left(\operatorname{Ad}\left(g^{-1}\right)(A)\right)^{*} .^{17}$

A connection $\Gamma$ on $P(M, G)$ associates a horizontal subspace $H_{p}<T_{p}(P)$ with every $p \in P$ such that

(1) $T_{p}(P)=V_{p} \oplus H_{p}$ with projections $v_{p}: T_{p}(P) \rightarrow V_{p}, h_{p}: T_{p}(P) \rightarrow H_{p}$;

(2) horizontal and vertical projections of vector fields exist with:

$$
\begin{aligned}
& v: \mathscr{D}^{\mathrm{d}}(P) \rightarrow v \mathscr{\mathscr { P }}^{\mathrm{d}}(P)=v\left(\mathscr{D}^{\mathrm{d}}(P)\right) \subseteq \mathscr{D}^{\mathrm{d}}(P), \\
& X \mapsto v X,(v X)(p):=v_{p} X(p), \\
& h: \mathscr{Q}^{\mathrm{I}}(P) \rightarrow h \mathscr{D}^{\mathrm{i}}(P):=h\left(\mathscr{D}^{\mathrm{1}}(P)\right) \subseteq \mathscr{D}^{\mathrm{d}}(P), \\
& X \mapsto h X,(h X)(p):=h_{p} X(p) ;
\end{aligned}
$$

(3) $\forall p \in P, \forall g \in G:\left(R_{g}\right)_{\star}\left(H_{p}\right)=H_{p \triangleright g}$.

The horizontal subspaces $H_{p}$ form the horizontal bundle $I I(P)$ with $T(P)=V(P) \oplus H(P)$; $\gamma(P(M, G))$ denotes the set of all connections on $P(M, G)$.

Every connection $\Gamma$ on a principal bundle $P$ defines a connection 1 -form $\omega^{\Gamma} \in \mathscr{B}_{1}(P, \mathfrak{g})$ by

$$
\omega^{\Gamma}(X)(p)=\omega^{\Gamma}(v X)(p)=\left(d \tau_{p}\right)^{-1}\left(v_{p} X(p)\right) \forall X \in \mathscr{D}^{1}(P) .
$$

For our purposes we concentrate on $\mathfrak{g} \cong \mathbb{R}$, so every definition for $\mathscr{A}(P)$ implies $\mathscr{A}(P, \mathfrak{g})$. Then $\omega^{\Gamma}\left(h \mathscr{P}^{\AA}(P)\right)=0, \omega^{\Gamma}\left(A^{*}\right)=A$ and $R_{g}^{\star} \omega^{\Gamma}=\omega^{\Gamma}$ for all $g \in G$. Vice versa, then every $\omega \in \mathscr{B}_{y}(P(M, G))$

$$
:=\left\{\omega \in \mathscr{B}_{1}(P, \mathfrak{g}) \mid \omega\left(A^{*}\right)=A \forall A \in \mathfrak{g}, R_{g}^{\star} \omega=\omega \forall g \in G\right\}
$$

defines a connection $\Gamma \in \gamma(P(M, G))$ by $\omega\left(h \mathscr{L}^{\mathrm{i}}(P)\right)=0$, and then $\omega=\omega^{\mathrm{\Gamma}}$.

For any $\omega_{s} \in \mathscr{B}_{s}(P)$, we define horizontal and vertical projections $\omega_{s} h \in \mathscr{B}_{s}(P) h$, resp., $\omega_{s} v \in \mathscr{A}_{s}(P) v$ by

$$
\omega_{s} h\left(X^{(1)}, \ldots, X^{(s)}\right)=\omega_{s}\left(h X^{(1)}, \ldots, h X^{(s)}\right),
$$




$$
\omega_{s} v\left(X^{(1)}, \ldots, X^{(s)}\right)=\omega_{s}\left(v X^{(1)}, \ldots, v X^{(s)}\right)
$$

for all vector fields $X^{(i)}$ in $D^{1}(P)$. Forms $\varphi$ that are invariant under $R_{g}$, i.e. $R_{g}^{\star} \varphi=\varphi$, are called pseudotensorial, their set is denoted by $\mathscr{A}^{P}(P(M, G))$, whereas $\mathscr{A}^{T}(P(M, G))$ means the set of all tensorial forms, i.e. of all horizontal pseudotensorial forms. $\pi^{\star}: \mathscr{A}(M) \rightarrow \mathscr{A}^{T}(P(M, G)$ ) is an isomorphism of $C^{\infty}(M)$-modules and the definitions of $A_{s}(P) h, \mathscr{C}^{P}(P(M, G))$ and $\mathscr{B}^{T}(P(M, G))$ are independent of the choice of a special connection. For all $r \in \mathbf{N}$ the forms $\left(\pi^{\star} d x_{\alpha}^{i_{1}}\right) \wedge \ldots \wedge\left(\pi^{\star} d x_{\alpha}^{i_{r}}\right) \cong d x_{\alpha}^{i_{1}} \wedge \ldots \wedge d x_{\alpha}^{i_{r}}$ are a local basis for $A_{r}^{T}\left(\pi^{-1}\left(U_{\alpha}\right)\right)$, resp., for $A_{r}\left(\pi^{-1}\left(U_{\alpha}\right)\right) h$ if the coefficient functions depend not only on $x \in U_{\alpha}$ but on $p \in \pi^{-1}\left(U_{\alpha}\right)$.

Lemma III.2: If $\Gamma \in \gamma(P(M, G)) \neq \varnothing$ then:

(1) $\omega^{\Gamma} \in \mathscr{H}_{\gamma}(P(M, G)) \subset \mathscr{B}_{1}^{P}(P(M, G)), \quad \omega^{\Gamma} h=0$;

(2) $\forall \varphi \in \mathscr{B}_{r}^{P}(P(M, G)), r>0: \varphi h \in \mathscr{B}_{r}^{T}(P(M, G))$;

(3) $\forall \varphi \in \mathscr{A}_{r}^{P}(P(M, G)): d \varphi \in \mathscr{B}_{r+1}^{P}(P(M, G))$.

The covariant exterior derivation $d^{\Gamma}: \mathscr{A}(P) \rightarrow \mathscr{A}(P) h$ is defined by $d^{\Gamma} \varphi_{r}:=\left(d \varphi_{r}\right) h$; $\Omega^{\Gamma}:=d^{\Gamma} \omega^{\Gamma} \in \mathscr{A}_{2}^{T}(P(M, G))$ is called curvature 2 -form for $\Gamma$. Using $d^{\Gamma} \circ R_{g}^{\star}=R_{g}^{\star} \circ d^{\Gamma}$-i.e., $d^{\Gamma}\left(\mathscr{G}_{r}^{P}(P(M, G))\right) \subseteq \mathscr{A}_{r+1}^{T}(P(M, G))$-as well as $d^{\Gamma} \circ \pi^{\star}=\pi^{\star} \circ d$, one shows

Lemma III.3: If $\mathfrak{g} \cong \mathrm{R}$ and $\omega^{\Gamma} \in \mathscr{A}_{\gamma}(P(M, G))$ then

$$
\begin{gathered}
\forall \varphi \in \mathscr{A}^{T}(P(M, G)): \quad d^{\Gamma} \varphi=d \varphi,\left(d^{\Gamma}\right)^{m} \varphi=0, m \geqslant 2 ; \\
\forall \alpha \in \mathscr{B}^{P}(P(M, G)): \quad\left(d^{\Gamma}\right)^{m} \alpha=0, m \geqslant 3 ; \\
\text { structure equation: } \quad \Omega^{\Gamma}=d^{\Gamma} \omega^{\Gamma}=d \omega^{\Gamma} ; \\
\text { Bianchi identity: } \quad d^{\Gamma} \Omega^{\Gamma}=d \Omega^{\Gamma}=0 .
\end{gathered}
$$

. Via the local sections $\sigma_{\alpha, e}, \omega^{\Gamma}$ and $\Omega^{\Gamma}$ [restricted to $\left.\pi^{-1}\left(U_{\alpha}\right)\right]$ define forms on the base

$$
\begin{gathered}
A^{\alpha}=A_{i}^{\alpha} d x^{i}:=\sigma_{\alpha, e}^{\star}\left(\omega^{\Gamma}\right) \in \mathscr{A}_{1}\left(U_{\alpha}, \mathfrak{g}\right), \\
F^{\alpha}=\frac{1}{2} F_{i j}^{\alpha} d x^{i} \wedge d x^{j}:=\sigma_{\alpha, e}^{\star}\left(\Omega^{\Gamma}\right) \in \mathscr{H}_{2}\left(U_{\alpha}, \mathfrak{g}\right),
\end{gathered}
$$

for which the following theorem holds (we write the group operation in $G$ additively):

Theorem III.4: Let $\mathfrak{g} \cong \mathrm{R}, \omega^{\Gamma} \in \mathscr{A}_{\gamma}(P(M, G))$ and $\left\{\left(U_{\alpha}, \psi_{\alpha}\right)\right\}_{\alpha \in A}$ a bundle atlas for $P$, then for all $\alpha, \beta \in A$ with $U_{\alpha \beta}:=U_{\alpha} \cap U_{\beta} \neq \varnothing$ and for all $x \in U_{\alpha \beta}$ :

$$
\begin{gathered}
F^{\alpha}=d A^{\alpha}, \quad d F^{\alpha}=0, \\
\left.A^{\alpha}\right|_{U_{\alpha \beta}}=\left.A^{\beta}\right|_{U_{\alpha \beta}}+d g_{\beta \alpha}, \quad A_{i}^{\alpha}(x)=A_{i}^{\beta}(x)+\partial_{i} g_{\beta \alpha}(x), \\
\left.F^{\alpha}\right|_{U_{\alpha \beta}}=\left.F^{\beta}\right|_{U_{\alpha \beta}}, \quad F_{i j}^{\alpha}(x)=F_{i j}^{\beta}(x) .
\end{gathered}
$$

Vice versa, if for a bundle atlas $\left\{\left(U_{\alpha}, \psi_{\alpha}\right)\right\}_{\alpha \in A}$ on $P(M, G)$ with $\mathfrak{g} \cong \mathbf{R}$ a family $\left\{A^{\alpha} \in \mathscr{B}_{1}\left(U_{\alpha}, \mathfrak{g}\right)\right\}_{\alpha \in A}$ is given such that (17) holds, then there exists one unique $\omega^{\Gamma} \in \mathcal{A}_{\gamma}(P(M, G))$ such that $A^{\alpha}=\sigma_{\alpha, e}^{\star}\left(\left.\omega^{\Gamma}\right|_{\pi^{-1}\left(U_{\alpha}\right)}\right)$ for all $\alpha \in A$.

If $M$ is pseudo-Riemannian, we can use its metric for horizontal forms on the bundle. For all $\alpha_{r}, \beta_{r} \in \mathscr{A}_{r}(P) h$ where locally $\left.\alpha_{r}\right|_{U_{\alpha}}=(1 / r !) \alpha_{i_{1} \ldots i_{r}}(p) d x_{\alpha}^{i_{1}} \wedge \ldots \wedge d x_{\alpha}^{i_{r}}$ and where $\left.\beta_{r}\right|_{U_{\alpha}}=(1 / r !) \beta_{i_{1}} \ldots i_{r}(p) d x_{\alpha}^{i_{1}} \wedge \ldots \wedge d x_{\alpha}^{i_{r}}$, we define, independently of the special trivialization, 


$$
\left\langle\alpha_{r}, \beta_{r}\right\rangle_{P}:=\frac{1}{r !} \alpha_{i_{1} \cdots i_{r}} \beta^{i_{1} \cdots i_{r}} \quad \forall p \in \pi^{-1}\left(U_{\alpha}\right) .
$$

Then, $R_{g}^{\star}\left(\left\langle\alpha_{r}, \beta_{r}\right\rangle_{P}\right)=\left\langle R_{g}^{\star} \alpha_{r}, R_{g}^{\star} \beta_{r}\right\rangle_{P}$ as well as $\left\langle\pi^{\star} \alpha_{r}, \pi^{\star} \beta_{r}\right\rangle_{P}=\left\langle\alpha_{r}, \beta_{r}\right\rangle$ for all $\alpha_{r}$, $\beta_{r} \in \mathscr{C}_{r}(M)$. For oriented $M$ we can thus define the Hodge star operator ${ }_{h}$ for horizontal forms $\beta_{r} \in \mathscr{\ell}_{r}(P) h$ by requiring

$$
\alpha_{r} \wedge{ }_{h} \beta_{r}=\left\langle\alpha_{r}, \beta_{r}\right\rangle_{P}\left(\pi^{\star} d V\right) \quad \forall \alpha_{r} \in \mathscr{H}_{r}(P) h,
$$

and get a Hodge star operator $* \Gamma: \mathscr{A}(P) \rightarrow \mathscr{A}(P) h$ via $* \Gamma \beta_{r}:={ }^{*}{ }_{h}\left(\beta_{r} h\right)$. This finally leads to the definition of the co-derivation $\delta^{\Gamma}$ and the Laplace-Beltrami operator $\Delta^{\Gamma}$ for a connection $\Gamma$ on a principal bundle $(n=\operatorname{dim} M)$ :

$$
\delta^{\Gamma} \varphi_{r}=-(-1)^{n(r-1)} *^{\Gamma} d^{\Gamma} *^{\Gamma} \varphi_{r}, \quad \Delta^{\Gamma} \varphi_{r}=\left(d^{\Gamma} \delta^{\Gamma}+\delta^{\Gamma} d^{\Gamma}\right) \varphi_{r}
$$

With regard to our purposes we get the following lemma:

Lemma III.5: For any connection $\Gamma$ on $P(M, G)$ and $\forall g \in G, \delta^{\Gamma}$ and $\Delta^{\Gamma}$ commute with $R_{g}^{\star} ;$ if $\mathfrak{g} \cong \mathrm{R}$ their operation on $A_{r}^{T}(P)$ does not depend on $\Gamma$ and we have

$$
\begin{gathered}
\left(\delta^{\Gamma}\right)^{m} \varphi_{r}=0, \quad m \geqslant 2, \quad \forall \varphi_{r} \in \mathscr{C}_{r}^{P}(P(M, G)) ; \\
\delta^{\Gamma_{\circ}} \pi^{\star}=\pi^{\star} \delta, \quad \Delta^{\Gamma \circ \pi^{\star}}=\pi^{\star} \circ \Delta ; \\
\delta^{\Gamma} \omega^{\Gamma}=0, \quad \Delta^{\Gamma} \omega^{\Gamma}=\delta^{\Gamma} \Omega^{\Gamma} .
\end{gathered}
$$

\section{Connections on associated bundles}

Every connection $\Gamma$ on a principal bundle $P(M, G)$ induces splittings $T(B)=H(B)$ $\oplus V(B)$ on any associated bundle $B(M, F, G)=P \times{ }_{G} F$. Let $q: P \times F \rightarrow B$ be the natural projection then $H(B):=q_{\star}(H(P) \times\{0\})$. Locally let $i_{f}(x, g)=(x, g, f)$ denote the inclusion and $q(x, g, f)=(x, g \triangleleft f)$. Then for $b=(x, f)$ we have

$$
H_{b}=d q_{(x, e, f)}\left(d i_{f}\right)_{(x, e)} H_{(x, e)}=d q_{\left(x, g, g^{-1} \triangleleft f\right)}\left(d i_{g^{-1} \triangleleft f}\right)_{(x, g)} H_{(x, g)} \quad \forall g \in G .
$$

If $\tau_{f}: G \rightarrow F: g \mapsto g \triangleleft f$ denotes the left action of $G$ on $F$ and $\partial_{i}-A_{i}^{g} \partial_{g} \in H_{(x, e)}$ with $A_{i}^{g} \in \mathbb{R}$ then $D_{i}=\partial_{i}-A_{i}^{g} d \tau_{f}\left(\partial_{g}\right)=: \partial_{i}+\tilde{A}_{i}^{j}\left(\partial / \partial y^{j}\right)$ is in $H_{(x, f)}$ (where $y^{j}, j=1, \ldots, \operatorname{dim} F$ is a local base for $F$ ). $D_{i}$ is called the horizontal lift of $\partial_{i}$ and from $A_{i}^{g} \partial_{g}-A^{\alpha}\left(\partial_{i}\right)=v_{(x, e)} \partial_{i}$ we have

$$
\begin{gathered}
h \partial_{i}=D_{i}=\partial_{i}-d \tau_{f}\left(A^{\alpha}\left(\partial_{i}\right)\right), \quad h \partial_{y j}=0, \\
v \partial_{i}=d \tau_{f}\left(A^{\alpha}\left(\partial_{i}\right)\right)=A_{i}^{g} d \tau_{f}\left(\partial_{g}\right), \quad v \partial_{y^{j}}=\partial_{y}, \\
h M^{\alpha}=M^{\alpha}-d \tau_{f}\left(A^{\alpha}\left(M^{\alpha}\right)\right), \quad h F^{\alpha}=0, \\
v M^{\alpha}=d \tau_{f}\left(A^{\alpha}\left(M^{\alpha}\right)\right), \quad v F^{\alpha}=F^{\alpha},
\end{gathered}
$$

for $M^{\alpha} \in T_{x}(M)$ and $F^{\alpha} \in T_{f}(F)$. The corresponding local projections of forms defined analogously to (13) and (14) are

$$
\begin{gathered}
d x^{i} v=0, \quad\left(d y^{j}\right) v=d y^{j}-\tilde{A}_{i}^{j} d x^{i}, \quad \text { resp., } \\
\mu_{(x, f)}^{\alpha} v=0, \quad \varphi_{(x, f)}^{\alpha} v=\varphi_{(x, f)}^{\alpha}+\left(\tau_{f}^{\star} \varphi_{(x, f)}^{\alpha}\right) \circ A_{x}^{\alpha},
\end{gathered}
$$


for 1 -forms $\omega^{\alpha}=\mu^{\alpha}+\varphi^{\alpha}$ with $\mu_{(x, f)}^{\alpha}: T_{x}(M) \rightarrow V$ and $\varphi_{(x, f)}^{\alpha}: T_{f}(F) \rightarrow V$. We verify that $v, h: \mathscr{P}^{A}(B) \rightarrow \mathscr{Z}^{1}(B)$ by checking the transformation rules for vector fields $Y \in \mathscr{D}^{1}(B)$ where $\left.Y\right|_{\pi^{-1}\left(U_{\alpha}\right)}\left(x, f^{\alpha}\right)=M_{\left(x, f^{\alpha}\right)}^{\alpha}+F_{\left(x, f^{\alpha}\right)}^{\alpha} \in T_{x}(M) \oplus T_{f^{\alpha}}(F):$ for all $x \in U_{\alpha \beta}$

$$
M_{\left(x, f^{\alpha}\right)}^{\alpha}=M_{\left(x, f^{\beta}\right)}^{\beta} \quad \text { and } \quad F_{\left(x, f^{\alpha}\right)}^{\alpha}=d L_{g_{\alpha \beta}(x)}\left(F_{\left(x, f^{\beta}\right)}^{\beta}\right)+d \tau_{f}^{\beta} d g_{\alpha \beta}\left(M_{\left(x, f^{\beta}\right)}^{\beta}\right)
$$

This corresponds to the following transformation rule for $V$-valued 1-forms $\omega \in \mathscr{A}_{1}(B, V)$ with $\left.\omega\right|_{\pi^{-1}\left(U_{\alpha}\right)}\left(x, f^{\alpha}\right)=\mu_{\left(x, f^{\alpha}\right)}^{\alpha}+\varphi_{\left(x, f^{\alpha}\right)}^{\alpha} \in \operatorname{Hom}\left(T_{x}(M), V\right) \oplus \operatorname{Hom}\left(T_{f^{\alpha}}(F), V\right)$ :

$$
\begin{gathered}
\varphi_{\left(x, f^{\alpha}\right)}^{\alpha}=L_{g_{\beta \alpha}(x)}^{\star} \varphi_{\left(x, f^{\beta}\right)}^{\beta} \quad \text { for all } x \in U_{\alpha \beta}, \\
\mu_{\left(x, f^{\alpha}\right)}^{\alpha}=\mu_{\left(x, f^{\beta}\right)}^{\beta}+g_{\beta \alpha}^{\star} \tau_{f^{\alpha}}^{\star} \varphi_{\left(x, f^{\beta}\right)}^{\beta}=\mu_{\left(x, f^{\beta}\right)}^{\beta}-\left(\tau_{f^{\beta}}^{\star} \varphi_{\left(x, f^{\beta}\right)}^{\beta}\right) \circ\left(g_{\alpha \beta}^{\star} \Theta_{G}^{L}\right)_{x} .
\end{gathered}
$$

For abelian $G$ with $L_{g}$ from (11) we have $\left(L_{g}\right)_{\star} H_{b}=H_{g \triangleleft b}$ and $\left(L_{g}^{\star} \varphi\right) v=L_{g}^{\star}(\varphi v)$, etc. As for the interplay between the transformation rules for differential forms and their vertical projections in the case $\mathfrak{g} \cong \mathbf{R}$, equations (17), (21), and (23) yield the following theorem:

Theorem III.6: Let $\Gamma$ be a connection on $P(M, G)$ where $G$ is abelion with $\mathfrak{g}=E \mathbf{R} \cong \mathbf{R}$, $B(M, F, G)$ an associated bundle and $V$ any vector space. For any $\chi \in \mathcal{A}_{n}(F, V)$ with $L_{8}^{\star} \chi=\chi$ for all $g \in G$ define $\nu \in \mathscr{B}_{n-1}(F, V)$ by

$$
\nu_{f}\left(F_{f}^{(1)}, \ldots, F_{f}^{(n-1)}\right):=n \cdot \chi_{f}\left(d \tau_{f}(E), F_{f}^{(1)}, \ldots, F_{f}^{(n-1)}\right)
$$

for all $f \in F$ and $F^{(i)} \in \mathscr{D}^{\mathrm{l}}(F)$. For any $U_{\alpha} \in \mathfrak{U}$ denote $\chi^{\alpha}:=\pi_{\alpha}^{\star} \chi, \nu^{\alpha}:=\pi_{\alpha}^{\star} \nu$. Then on all $U_{\alpha \beta} \neq \varnothing$

$$
\begin{gathered}
\chi^{\alpha}=\chi^{\beta}+\frac{1}{E} d g_{\alpha \beta} \wedge \nu \\
\chi^{\alpha} v=\chi^{\alpha}+\frac{1}{E} A^{\alpha} \wedge \nu=\chi^{\beta}+\frac{1}{E} A^{\beta} \wedge \nu=\chi^{\beta} v \\
\nu^{\alpha}=\nu^{\alpha} v=\nu^{\beta}=\nu^{\beta} v
\end{gathered}
$$

Thus $\chi v$ and $\nu$ define global vertical $V$-valued forms on $B$, that are invariant under $L_{g}^{\star}$.

Finally, if $B$ is a vector bundle over $M$, every connection $\Gamma$ defines covariant derivatives of sections $\sigma: M \rightarrow B$. Every vector field $X=X^{i} \partial_{i} \in \mathscr{D}^{\lambda}(M)$ naturally defines a function $\sigma_{\star} X: M \rightarrow T(B): x \mapsto X^{i} \partial_{i} \sigma(x) \in T_{\sigma(x)}(B)$, and by projection onto the vertical bundle we get a map $v\left(\sigma_{\star} X\right): M \rightarrow V(B)$. Now if $B$ is a vector bundle we can identify the fiber and its tangential space and thus $v\left(\sigma_{\star} X\right)$ defines a section $\nabla_{X} \sigma: M \rightarrow B$, the covariant derivative of $\sigma$ in the direction of $X$. If locally $\sigma^{\alpha}(x)=\left(x, f^{\alpha}(x)\right)$ then $\nabla_{X}^{\alpha} \sigma^{\alpha}=\left(\right.$ id, $\left.\nabla_{X}^{\alpha} f^{\alpha}\right)$ with

$$
\nabla_{X}^{\alpha} f^{\alpha}:=X\left(f^{\alpha}\right)+d \tau_{f^{\alpha}}\left(A^{\alpha}(X)\right)
$$

e.g.:

$$
\nabla_{i}^{\alpha} f^{\alpha}(x):=\partial_{i} f^{\alpha}(x)+d \tau_{f^{\alpha}}\left(A^{\alpha}\left(\partial_{i}\right)\right)
$$

Thus under the left action of $G$ on $F$ and so under any change of the bundle chart the covariant derivatives $\nabla_{X}^{\alpha} f^{\alpha}$ transform in the same manner as the local sections $f^{\alpha}$ themselves. 
TABLE I. The Maxwell connection on $P\left(M, G_{\mathrm{em}}\right)$.

\begin{tabular}{lcc}
\hline \hline & $\mathrm{M}$ & $P\left(M, G_{\mathrm{em}}\right)$ \\
\hline gauge forms & $F=d A, F^{\alpha}=d A^{\alpha}$ & $\Omega^{\Gamma}=d^{\Gamma} \omega^{\Gamma}$ \\
homogeneous Maxwell equations & $d F=0$ & $d^{\Gamma} \Omega^{\Gamma}=0$ \\
inhomogeneous Maxwell equations & $\delta F=-\frac{4 \pi}{c} J$ & $\delta^{\Gamma} \Omega^{\Gamma}=-\frac{4 \pi}{c} J^{\Gamma}$ \\
& $\delta J=0$ & $\delta^{\Gamma} J^{\Gamma}=0$ \\
continuity equation & $\Delta F=0$ & $\Delta^{\Gamma} \Omega^{\Gamma}=0$ \\
$0=J=J^{\Gamma}$ yields: & $\delta A=0, \delta A^{\alpha}=0$ & $\delta^{\Gamma} \omega^{\Gamma}=0$ \\
Lorentz gauging & $\Delta A=-\frac{4 \pi}{c} J$ & $\Delta^{\Gamma} \omega^{\Gamma}=-\frac{4 \pi}{c} J^{\Gamma}$ \\
Lorentz gauging yields: &
\end{tabular}

\section{MONOPOLE AND SKYRMION BUNDLE}

Now the mathematical background is set for the description of the interaction between monopoles and baryons. For the electromagnetic fields we construct a principal bundle $P\left(M, G_{\mathrm{em}}\right)$ over space-time $M$. Gauge fields and electromagnetic fields on $M$ are combined with a Maxwell connection $\Gamma$ on $P\left(M, G_{\mathrm{em}}\right)$ and its forms $\omega^{\Gamma}$ and $\Omega^{\Gamma}$, from which the former are obtained by (15) and (16). In order to guarantee that all $F^{\alpha}$ are restrictions of a global real-valued 2-form $F$ (in opposite to the general case of Yang-Mills theories) the only possible choice of a connected Lie group $G_{\mathrm{em}}$ that admits nontrivial bundles is $G_{\mathrm{em}}=2 g_{D} \cdot \mathrm{S}^{1} \cong \mathrm{U}_{1}$. We thus have $\Omega^{\Gamma}=\pi^{\star} F$ and together with $J^{\Gamma}:=\pi^{\star} J, \delta^{\Gamma}$ and $\Delta^{\Gamma}$, the electromagnetic equations can be cxpressed as differential equations on the bundle, cf. Table $I$. Note that with our definition of $\delta^{\Gamma}, \omega^{\Gamma}$ is automatically "Lorentz gauged."

Locally $\left\{d x^{\mu}, \mu=0, \ldots, 3 ; \omega^{\alpha}=d g+A_{\mu}^{\alpha} d x^{\mu}\right\}$ and $\left\{\partial_{\mu}-A_{\mu}^{\alpha} \partial_{g}, \mu=0, \ldots, 3 ; \partial_{g}\right\}$ are the dual bases of $A_{1}\left(\pi^{-1}\left(U_{\alpha}\right)\right)$ and $\mathscr{D}^{1}\left(\pi^{-1}\left(U_{\alpha}\right)\right)$ adapted to $\Gamma$.

Whereas the Maxwell connection on $P$ is associated with $J$, the structure of $P$ itself is fixed by the number and distribution of magnetic (anti-)monopoles in the "holes" of $M$. In our case of a single monopole we have a bundle

$$
P\left(\mathbb{R}_{(t)} \times \mathbb{R}_{(r)}^{+} \times \mathrm{S}^{2}, G_{\mathrm{em}}\right) \cong P\left(\mathrm{~S}^{2}, G_{\mathrm{em}}\right) \times \mathbb{R}_{(t)} \times \mathbb{R}_{(r)}^{+},
$$

where $P\left(\mathrm{~S}^{2}, G_{\mathrm{em}}\right) \cong P\left(\mathrm{~S}^{2}, \mathrm{~S}^{1}\right)$ is the only topological interesting part. For these sphere bundles the following classification theorem holds: ${ }^{16}$

Theorem IV.I: If $G$ is arcwise connected, then the set of equivalence classes of bundles over $\mathrm{S}^{n}$ with group $G$ is in 1-1 correspondence with $\pi_{n-1}(G)$.

So there is a countable number of different principal bundles $P_{m}\left(\mathrm{~S}^{2}, \mathrm{~S}^{1}\right)$ with $m \in \mathbb{Z}$, showing the topological quantization of the magnetic charge $m g_{D}$, and we can put $2 e g_{D}=1$.

While $P_{0}\left(\mathrm{~S}^{2}, \mathrm{~S}^{1}\right)=\mathrm{S}^{2} \times \mathrm{S}^{1}$ describes the trivial case without monopoles, $P_{1}\left(\mathrm{~S}^{2}, \mathrm{~S}^{1}\right)=\mathrm{S}^{3}$ is the bundle for the single charged monopole. Here $\pi: S^{3} \rightarrow S^{2}$ is the Hopf fibering: the Lie group $\mathrm{S}^{3} \cong \mathrm{SU}_{2}$ contains $\mathrm{S}^{1}$ as a closed subgroup, so the base $\mathrm{S}^{2} \cong \mathrm{S}^{3} / \mathrm{S}^{1}$ represents the quotient manifold of equivalence classes under $\mathrm{S}^{1}$. For $m>1, \quad P_{m}\left(\mathrm{~S}^{2}, \mathrm{~S}^{1}\right)=\mathrm{S}^{3} / H_{m}$, where $H_{m}=\{(z / m) \cdot 2 \pi\}_{z \in Z_{m}} \cong Z_{m}=\mathbb{Z} / m Z$ are the only closed subgroups of $\mathbf{S}^{1}$. Finally all bundles $P_{-m}\left(\mathrm{~S}^{2}, \mathrm{~S}^{1}\right)$ with $m \geqslant 1$ are obtained out of their pendants $P_{m}$ by reflecting the base $\mathrm{S}^{2}$ at its equator.

For all $P_{m}\left(\mathrm{~S}^{2}, \mathrm{~S}^{1}\right)$ local trivializations exist over the northern and the southern hemisphere $U_{+}$, resp., $U_{-}$. The transition function $g_{-+}=-g_{+-}$is

$$
g_{-+}: U_{+} \cap U_{-} \rightarrow \mathrm{S}^{1}:(\theta, \phi) \mapsto m \phi
$$


and thus for all $p_{m} \in P_{m}$ with $\pi\left(p_{m}\right) \in U_{+} \cap U_{-}$and $p_{m} \cong\left(\pi\left(p_{m}\right), \varphi_{m}^{(+)}\right) \cong\left(\pi\left(p_{m}\right), \varphi_{m}^{(-)}\right)$we obtain $\varphi_{m}^{(-)} \equiv \varphi_{m}^{(+)}+m \phi(\bmod 2 \pi)$. In accordance with $(10)$, equations $(17)$ and $(18)$ thus yield

$$
A^{(+)}=A^{(-)}+2 m g_{D} d \phi, \quad F^{(+)}=F^{(-)} .
$$

Next for the skyrmions: instead of considering functions $U: M \rightarrow \mathrm{SU}_{n}$ we can equivalently think of the meson fields as sections in $M \times S_{n}$. This allows for the description of the interaction with electromagnetic fields: ${ }^{3}$ meson fields are considered as global sections in an associated bundle $B\left(M, \mathrm{SU}_{n}, G_{\mathrm{em}}\right)$. The left action of $G_{\mathrm{em}}$ on $\mathrm{SU}_{n}$ is given by the inner automorphisms

$$
g \triangleleft U=\tau_{U}(g)=L_{g}(U)=e^{-i e g Q} U e^{+i e g Q},
$$

which do not affect the vacuum state being diagonal symmetry operations. $Q$ is the hermitian $n \times n$-matrix containing the quark charges in units of $e$ (again $n=N_{F}=2$, resp., 3)

$$
Q=\left(\begin{array}{cc}
\frac{2}{3} & 0 \\
0 & -\frac{1}{3}
\end{array}\right), \quad \text { resp., } \quad Q=\left(\begin{array}{ccc}
\frac{2}{3} & 0 & 0 \\
0 & -\frac{1}{3} & 0 \\
0 & 0 & -\frac{1}{3}
\end{array}\right)
$$

From a physical point of view it is obvious that any coupling between baryons and electromagnetic fields has to involve these charges. From a mathematical point of view we observe that $Q$ 's eigenvalues $\lambda_{i} \in \mathbf{R}$ obey the conditions $\lambda_{i}-\lambda_{j} \in \mathbf{Z}$ and $\operatorname{gcd}\left\{\lambda_{i}-\lambda_{j}\right\}=1$, which guarantee that the action is well defined and effective. The transition functions are

$$
U^{\alpha}(x)=e^{-i e g_{\alpha \beta}(x) Q} U^{\beta}(x) e^{+i e g_{\alpha \beta}(x) Q},
$$

resp.,

$$
U^{(-)}(x)=e^{-i m \phi Q} U^{(+)}(x) e^{+i m \phi Q}
$$

for the skyrmion bundle $B_{m}\left(M, \mathrm{SU}_{n}, G_{\mathrm{em}}\right)$ associated with $P_{m}$. So not only vacuum $U \equiv 1$ is a global section but every $U(x)=e^{i \chi(x) Q}$ with a differentiable map $\chi: M \rightarrow \mathrm{S}^{1}$.

Let us see how the local representations of forms transform under a change of bundle charts. From (26) we get

$$
\begin{gathered}
d U^{\alpha}=g_{\alpha \beta} \triangleleft\left(d U^{\beta}-i e d g_{\alpha \beta}\left[Q, U^{\beta}\right]\right), \\
L^{\alpha}=g_{\alpha \beta} \triangleleft\left[L^{\beta}-i e d g_{\alpha \beta}\left(U^{\dagger}[Q, U]\right)^{\beta}\right], \\
R^{\alpha}=g_{\alpha \beta} \triangleleft\left[R^{\beta}-i e d g_{\alpha \beta}\left([Q, U] U^{\dagger}\right)^{\beta}\right], \\
\left(L^{k}\right)^{\alpha}=g_{\alpha \beta} \triangleleft\left\{\left(L^{k}\right)^{\beta}+i e d g_{\alpha \beta} \wedge \sum_{j=1}^{k}(-1)^{j}\left(L^{j-1} \wedge\left(U^{\dagger}[Q, U]\right) L^{k-j}\right)^{\beta}\right\}, \\
\left(R^{k}\right)^{\alpha}=g_{\alpha \beta} \triangleleft\left\{\left(R^{k}\right)^{\beta}+i e d g_{\alpha \beta} \wedge \sum_{j=1}^{k}(-1)^{j}\left(R^{j-1} \wedge\left([Q, U] U^{\dagger}\right) R^{k-j}\right)^{\beta}\right\},
\end{gathered}
$$

using $d g_{\alpha \beta} \wedge d g_{\alpha \beta}=0$. One easily proves the following lemma:

Lemma IV.2: $\rho_{1}^{Q}+\lambda_{1}^{Q}$ and $\rho_{2 l}^{Q}-\lambda_{2 l}^{Q}$ for $l \in \mathrm{N}_{0}$ are global forms on $B$, we have:

$$
\left(\rho_{2 l}^{Q}-\lambda \frac{Q}{2 l}\right)^{\alpha}=\left(\rho_{2 l}^{Q}-\lambda_{2 l}^{Q}\right)^{\beta}, \quad\left(\rho_{1}^{Q}+\lambda_{1}^{Q}\right)^{\alpha}=\left(\rho_{1}^{Q}+\lambda_{1}^{Q}\right)^{\beta},
$$




$$
\begin{aligned}
& \left(\rho_{3}^{Q}+\lambda_{3}^{Q}\right)^{\alpha}=\left(\rho_{3}^{Q}+\lambda \frac{Q}{3}\right)^{\beta}-2 i e d g_{\alpha \beta} \wedge \operatorname{Tr}\left[Q^{2}\left(R^{2}-L^{2}\right)+Q d U^{\dagger} \wedge Q d U\right]^{\alpha / \beta}, \\
& \left(\rho_{2 l+1}^{Q}+\lambda \frac{Q}{2 l+1}\right)^{\alpha}=\left(\rho_{2 l+1}^{Q}+\lambda \frac{Q}{2 l+1}\right)^{\beta}-2 i e d g_{\alpha \beta} \wedge \sum_{j=1}^{l} \operatorname{Tr}\left(Q U L^{2 j-1} Q L^{2 l-2 j+1} U^{\dagger}\right)^{\alpha / \beta} \\
& -i e d g_{\alpha \beta} \wedge \sum_{j=0}^{l} \operatorname{Tr}\left(Q R^{2 j} Q R^{2 l-2 j}-Q L^{2 j} Q L^{2 l-2 j}\right)^{\alpha / \beta} \\
& \omega_{2 l+1}^{\alpha}=\omega_{2 l+1}^{\beta}-(2 l+1) i e d g_{\alpha \beta} \wedge\left(\rho_{2 l}^{Q}-\lambda \frac{Q}{2 l}\right), \\
& \omega_{l_{1}}^{\alpha} \wedge \ldots \wedge \omega_{l_{k}}^{\alpha}=\omega_{l_{1}}^{\beta} \wedge \ldots \wedge \omega_{l_{k}}^{\beta}+\sum_{j=1}^{k}(-1)^{j} l_{j} i \text { e } d g_{\alpha \beta} \wedge \omega_{l_{1}}^{\alpha / \beta} \wedge \ldots \wedge \omega_{l_{j-1}}^{\alpha / \beta} \\
& \wedge\left(\rho_{l_{j}-1}^{Q}-\lambda l_{l_{j}-1}^{Q}\right) \wedge \omega_{l_{j+1}}^{\alpha / \beta} \wedge \cdots \wedge \omega_{l_{k}}^{\alpha / \beta}
\end{aligned}
$$

for any $U_{\alpha \beta}=U_{\alpha} \cap U_{\beta} \neq \varnothing$, if $l_{j}=2 n_{j}+1$ odd for $j=1, \ldots, k . \alpha / \beta$ indicates that one may use both trivializations because of the factor $d g_{\alpha \beta}$.

Now we are looking for the connection that $\Gamma$ induces on $B: T_{U}\left(\mathrm{SU}_{n}\right)=U \cdot \mathfrak{s u}_{n}$

$$
=\left\{H \in \mathrm{C}^{n \times n} \mid \operatorname{Tr}\left(U^{\dagger} H\right)=0 \wedge H^{\dagger}=-U^{\dagger} H U^{\dagger}\right\}
$$

and for the differential $d \tau_{U}: 2 g_{D} \mathrm{R} \rightarrow T_{U}\left(\mathrm{SU}_{n}\right)$ we get $d \tau_{U}(A)=-i e A[Q, U]$. Evaluating (19) we thus have $D_{\mu}^{\alpha}=\partial_{\mu}+i e A_{\mu}^{\alpha}\left[Q, U^{\alpha}\right] \in H_{\left(x, U^{\alpha}\right)}$ as a local base for $H_{\left(x, U^{\alpha}\right)}$ [while $\left.V_{\left(x, U^{\alpha}\right)}=T_{U^{\alpha}}\left(\mathrm{SU}_{n}\right)\right]$, and for $X=X^{\mu} \partial_{\mu}+M^{\alpha}, M^{\alpha} \in T_{U^{\alpha}}\left(\mathrm{SU}_{n}\right)$ we obtain from (20), resp., (21)

$$
h X=X^{\mu} D_{\mu}^{\alpha}, \quad v X=X-h X=M^{\alpha}-i e X^{\mu} A_{\mu}^{\alpha}\left[Q, U^{\alpha}\right] .
$$

For the differential forms on $\mathrm{SU}_{n}$, (22) yields

$$
\left(d U^{\alpha}\right) h=\text { ie } A^{\alpha}\left[Q, U^{\alpha}\right]
$$

and

$$
\left(d U^{\alpha}\right) v=d U^{\alpha}-i e A^{\alpha}\left[Q, U^{\alpha}\right]
$$

finally for the covariant derivative of sections, treating $B\left(M, \mathrm{SU}_{n}, G_{\mathrm{em}}\right)$ as a subbundle of $B\left(M, \mathbb{C}^{n \times n}, G_{\mathrm{em}}\right)$, we get from (25)

$$
\nabla_{\mu}^{\alpha} U^{\alpha}=\partial_{\mu} U^{\alpha}-i e A_{\mu}^{\alpha}[Q, U]
$$

Equations (17) and (28) yield $\left(d U^{\alpha}\right) v=g_{\alpha \beta} \triangleleft\left(d U^{\beta}\right) v$, and so $\omega_{2 l+1} v, \rho_{l}^{Q} v$ and $\lambda_{l}^{Q} v$ are global forms on $B$ because of $\omega_{2 l+1}^{\alpha} v=\omega_{2 l+1}^{\beta} v$ etc., cf. Theorem III.6. This proves the following lemma:

Lemma IV.3: $\omega_{2 l+1} v, \rho_{l}^{Q} v$ and $\lambda_{l}^{Q} v$ for $l \in \mathbf{N}_{0}$ are global forms on $B$ and we have:

$$
\begin{gathered}
\omega_{2 l+1}^{\alpha} v=\omega_{2 l+1}^{\alpha}-(2 l+1) \text { ie } A^{\alpha} \wedge\left(\rho_{2 l}^{Q}-\lambda \frac{Q}{2 l}\right), \\
\left(\rho_{2 l}^{Q}\right)^{\alpha} v=\left(\rho_{2 l}^{Q}\right)^{\alpha}-i e A^{\alpha} \wedge \sum_{j=1}^{2 l}(-1)^{j} \operatorname{Tr}\left(Q U L^{j-1} Q L^{2 l-j} U^{\dagger}\right)^{\alpha} \\
\left(\lambda_{2 l}^{Q}\right)^{\alpha} v=\left(\lambda_{2 l}^{Q}\right)^{\alpha}-i e A^{\alpha} \wedge \sum_{j=1}^{2 l}(-1)^{j} \operatorname{Tr}\left(Q U L^{j-1} Q L^{2 l-j} U^{\dagger}\right)^{\alpha}
\end{gathered}
$$




$$
\begin{gathered}
\left(\rho_{2 l+1}^{Q}\right)^{\alpha} v=\left(\rho_{2 l+1}^{Q}\right)^{\alpha}-i e A^{\alpha} \wedge \sum_{j=1}^{2 l+1} \operatorname{Tr}\left(Q R^{j-1} Q R^{2 l+1-j}-Q L^{j-1} U^{\dagger} Q U L^{2 l+1-j}\right)^{\alpha}, \\
\left(\lambda_{2 l+1}^{Q}\right)^{\alpha} v=\left(\lambda_{2 l+1}^{Q}\right)^{\alpha}-i e A^{\alpha} \wedge \sum_{j=1}^{2 l+1} \operatorname{Tr}\left(Q U L^{j-1} Q L^{2 l+1-j} U^{\dagger}-Q L^{j-1} Q L^{2 l+1-j}\right)^{\alpha}, \\
\left(\rho_{2 l}^{Q}-\lambda_{2 l}^{Q}\right) v=\rho_{2 l}^{Q}-\lambda \frac{Q}{2 l}, \quad\left(\rho_{1}^{Q}+\lambda_{1}^{Q}\right) v=\rho_{1}^{Q}+\lambda_{1}^{Q}, \\
\left(\rho_{3}^{Q}+\lambda_{3}^{Q}\right)^{\alpha} v=\left(\rho_{3}^{Q}+\lambda_{3}^{Q}\right)^{\alpha}-2 i e A^{\alpha} \wedge \operatorname{Tr}\left[Q^{2}\left(R^{2}-L^{2}\right)+Q d U^{\dagger} \wedge Q d U\right]^{\alpha}, \\
\left(\rho_{2 l+1}^{Q}+\lambda_{2 l+1}^{Q}\right)^{\alpha} v=\left(\rho_{2 l+1}^{Q}+\lambda_{2 l+1}^{Q}\right)^{\alpha}-2 i e A^{\alpha} \wedge \sum_{j=1}^{l} \operatorname{Tr}\left(Q U L^{2 j-1} Q L^{2 l-2 j+1} U^{\dagger}\right)^{\alpha} \\
-i e A^{\alpha} \wedge \sum_{j=0}^{l} \operatorname{Tr}\left(Q R^{2 j} Q R^{2 l-2 j}-Q L^{2 j} Q L^{2 l-2 j}\right)^{\alpha} .
\end{gathered}
$$

For calculations we need the action integral and the topological charge. Both consist of forms on $B$, whose pullbacks by the mesonic sections $U: M \rightarrow B$ are integrated over space-time, resp., the space manifold only. For the nonanomalous action our task is pretty simple now. Defining $\tilde{L}_{\mu}^{\alpha}:=\left(U^{\dagger} \nabla_{\mu} U\right)^{\alpha}$ by (29), we get for the lagrangian from (7) and (8)

$$
\begin{aligned}
\mathscr{L}_{N A}(A)= & -\frac{f_{\pi}^{2}}{4} \operatorname{Tr}\left(\tilde{L}_{\mu} \tilde{L}^{\mu}\right)+\frac{1}{32 a^{2}} \operatorname{Tr}\left(\left[\tilde{L}_{\mu}, \tilde{L}_{\nu}\right]\left[\tilde{L}^{\mu}, \tilde{L}^{\nu}\right]\right) \\
& +\frac{1}{32 f^{2}} \operatorname{Tr}\left(\left\{\tilde{L}_{\mu}, \tilde{L}_{\nu}\right\}\left\{\tilde{L}^{\mu}, \tilde{L}^{\nu}\right\}\right)+\frac{1}{32 g^{2}} \operatorname{Tr}\left(\nabla_{\mu} \tilde{L}_{\nu} \nabla^{\mu} \tilde{L}^{\nu}\right)
\end{aligned}
$$

where we omitted the index $\alpha$ because covariant derivation yields $\mathscr{L}_{N A}^{\alpha}=\mathscr{L}_{N A}^{\beta} \in C^{\infty}(B)$. A mass term may also be included $\left(\left[M_{g}, Q\right]=0\right)$. Combined with $\pi^{\star} d V \in \mathscr{C}_{4}(B) h$ we get $\Gamma_{N A}(U, A)$

$$
=\int_{M} U^{\star}\left(\mathscr{L}_{N A}(A) \pi^{\star} d V\right)=\int_{M} \mathscr{L}_{N A}(U, A) d V
$$

Next, for the anomalous action and the topological charge, a difficulty arises because we have to integrate over differential forms on the fiber. Whereas every form on $M$ (here $d V$ ) can be lifted onto $B$ by use of the pullback $\pi^{\star}$, there is no such mean for the forms $\omega_{3}$, resp., $\omega_{5} \in \mathscr{A}\left(\mathrm{SU}_{n}, \mathrm{C}\right)$. Only locally using $\pi_{\alpha}^{\star}$ we can inject $H^{*}\left(\mathrm{SU}_{n}, \mathrm{C}\right)$ into $H^{*}\left(\pi^{-1}\left(U_{\alpha}\right), \mathrm{C}\right)$ (cf. Lemma IV.2). Projection onto $\mathscr{f}(B, \mathrm{C}) v$ indeed produces global forms (Lemma IV.3), but in general they are not closed. Witten ${ }^{6,2}$ introduced generalized forms by "trial-and-error" and "little experimentation" and afterwards checked gauge invariance and closedness, but this has to be done for every form separately and says nothing about exactness nor uniqueness of these forms. Instead, we will examine the topology of the skyrmion bundle in general, mainly its homotopy and cohomology. For the latter we will use the results on spectral sequences from Bott and Tu. ${ }^{18}$ Aside from the fact that the topology of the skyrmion bundle is interesting out of itself, the examination of the cohomology $H^{*}(B)$ also shows how the closed forms on the fiber transform into closed forms on the bundle. Specialization to $\omega_{3}$ and $\omega_{5}$ and adaptation to $\Gamma$ will then yield the desired results. 
TABLE II. Homotopy groups of the skyrmion bundle $\pi_{k}\left(B_{m}\left(M, \mathrm{SU}_{n}, G_{\mathrm{em}}\right)\right)$.

\begin{tabular}{ccccccccccccccccccc}
\hline$n$ & $k=0$ & 1 & 2 & 3 & 4 & 5 & 6 & 7 & 8 & 9 & 10 & 11 & 12 & 13 & 14 & 15 \\
\hline 2 & 0 & 0 & $Z$ & $Z^{2}$ & $2^{2}$ & $2^{2}$ & $12^{2}$ & $2^{2}$ & $2^{2}$ & $3^{2}$ & $15^{2}$ & $2^{2}$ & $2^{4}$ & $6^{2}+4^{2}$ & $84^{2}+2^{4}$ & $2^{4}$ \\
3 & 0 & 0 & $\mathrm{Z}$ & $\mathrm{Z}^{2}$ & 2 & $\mathrm{Z}+2$ & $12+6$ & 2 & $12+2$ & $3^{2}$ & $15^{2}+2$ & $4+2$ & $60+2^{2}$ & $6^{2}+4$ & $84^{2}+2^{3}$ & $36+2^{2}$ \\
4 & 0 & 0 & $\mathrm{Z}$ & $\mathrm{Z}^{2}$ & 2 & $\mathrm{Z}+2$ & 12 & $\mathrm{Z}+2$ & $24+2$ & 6 & $15^{2}+8+2$ & $4+2$ & $60+2^{2}$ & $6+4^{2}$ & $1680+84+2^{3}$ & $72+2^{3}$ \\
5 & 0 & 0 & $\mathrm{Z}$ & $\mathrm{Z}^{2}$ & 2 & $\mathrm{Z}+2$ & 12 & $\mathrm{Z}+2$ & 2 & $\mathrm{Z}+3$ & $15^{2}+8$ & 2 & $360+2^{2}$ & $6+4^{2}$ & $1680+84+2^{2}$ & $3+2^{3}$ \\
6 & 0 & 0 & $\mathrm{Z}$ & $\mathrm{Z}^{2}$ & 2 & $\mathrm{Z}+2$ & 12 & $\mathrm{Z}+2$ & 2 & $\mathrm{Z}+3$ & 15 & $\mathrm{Z}+2$ & $720+2^{2}$ & $12+2^{2}$ & $5040+84+2^{3}$ & $3+2^{3}$ \\
\hline \hline
\end{tabular}

\section{TOPOLOGY OF THE SKYRMION BUNDLE}

\section{A. Homotopy groups}

Let us first concentrate on the homotopy groups of the skyrmion bundle. $B\left(M, \mathrm{SU}_{n}, G_{\mathrm{em}}\right)$ is arcwise connected since $M$ and $\mathrm{SU}_{n}$ are arcwise connected. So $\pi_{0}\left(B\left(M, \mathrm{SU}_{n}, G_{\mathrm{em}}\right)\right)=0$.

Every continuous map $f: M_{1} \rightarrow M_{2}$ induces group homomorphisms $f_{\star}: \pi_{n}\left(M_{1}\right) \rightarrow \pi_{n}\left(M_{2}\right)$ for all $n \in \mathrm{N}$. For any bundle $B(M, F)$ we have a "short exact sequence"

$$
\underset{0 \rightarrow F \rightarrow B}{i_{x}} \stackrel{\pi}{\rightarrow} M \rightarrow 0,
$$

where $i_{x}:=\left(\pi_{\alpha, x}\right)^{-1}$ is a diffeomorphism for any $x \in M$. This produces an infinite exact sequence of homotopy groups ${ }^{16}$

$$
\begin{gathered}
\ldots \rightarrow \pi_{n}(F) \stackrel{i \star}{\rightarrow} \underset{n}{\rightarrow} \pi_{n}(B) \stackrel{\pi_{\star}}{\rightarrow} \pi_{n}(M) \rightarrow \\
\rightarrow \pi_{n-1}(F) \stackrel{i_{\star}}{\rightarrow} \cdots \pi_{\star}^{\rightarrow} \pi_{0}(M) \rightarrow 0 .
\end{gathered}
$$

(If the manifolds fail to be groups, the last functions are just maps between sets.) Now if a global section $\sigma$ exists then $\pi_{\star}$ is surjective because $\pi_{\star} \circ \sigma_{\star}=\left(i d_{M}\right)_{\star}=i d$. So exactness of (31) yields that the long sequence splits into short ones, which proves the following direct sum theorem:

Theorem V.1: If a bundle $B(M, F)$ admits a section, then we have the direct sum relation

$$
\pi_{1}(B) \cong \pi_{1}(M) \ltimes \pi_{1}(F), \pi_{n}(B) \cong \pi_{n}(M) \oplus \pi_{n}(F), n \geqslant 2 .
$$

For direct products this implies $\pi_{n}\left(M_{1} \times M_{2}\right) \cong \pi_{n}\left(M_{1}\right) \oplus \pi_{n}\left(M_{2}\right), n \in \mathrm{N}_{0}$ [cf. (1) and (2), where $\left.\pi_{1}\left(\mathrm{~S}^{1}\right) \cong \mathrm{Z}, \pi_{n}\left(\mathrm{~S}^{1}\right)=0, n \neq 1\right]$. Now $\pi_{1}\left(\mathrm{SU}_{n}\right)=0$, so $\pi_{1}(B) \cong \pi_{1}(M)$. Because $M$ is simply connected-as long as the monopoles are isolated-Theorem V.1 thus yields $\pi_{1}\left(B\left(M, \mathrm{SU}_{n}, G_{\mathrm{em}}\right)\right)=0$, so $B$ is simply connected, too. This implies that $B$ is orientable because every simply connected manifold is orientable. ${ }^{18}$ We have proved:

Theorem V.2: The homotopy groups of the skyrmion bundle $B\left(M, \mathrm{SU}_{n}, G_{\mathrm{em}}\right)$ are independent of the monopole charges. They are isomorphic to the homotopy groups of the direct product $M \times \mathrm{SU}_{n}$ :

$$
\pi_{k}\left(B\left(M, \mathrm{SU}_{n}, G_{e m}\right)\right) \cong \pi_{k}(M) \oplus \pi_{k}\left(\mathrm{SU}_{n}\right), \quad k \in \mathrm{N}_{0} .
$$

As far as the homotopy groups of $M$ and $\mathrm{SU}_{n}$ are known, this gives the homotopy groups of the skyrmion bundle. As an example we have listed the homotopy groups for $B_{m}\left(M, \mathrm{SU}_{n}, G_{\mathrm{em}}\right)$ in Table II for $n=N_{F}=2,3, \ldots, 6$ using $\pi_{k}(M) \cong \pi_{k}\left(\mathrm{~S}^{2}\right)$ and $\pi_{k}\left(\mathrm{SU}_{n}\right)$ from Ref. 19. Here $\mathrm{Z}+2$ labels $\mathrm{Z} \oplus \mathrm{Z}_{2}$, etc. 


\section{B. Cohomology and spectral sequences}

In contrast to the homotopy groups, the cohomology of a bundle is more difficult to determine. Even for a direct product the cohomology groups $H^{k}\left(M_{1} \times M_{2}\right)$ are not just direct sums of $H^{k}\left(M_{1}\right)$ and $H^{k}\left(M_{2}\right)$ but are built up from these components according to the Künneth formula, which is slightly more complicated, especially in the case of the integer-valued cohomology $H^{*}(M, Z)$ :

Theorem V.3 (Künneth formula): If $M_{1}$ and $M_{2}$ are manifolds and if $H^{*}\left(M_{1}, Z\right)$ or $H^{*}\left(M_{2}, \mathrm{Z}\right)$ is finitely generated then

$$
\begin{aligned}
H^{p}\left(M_{1} \times M_{2}, \mathrm{Z}\right) \cong \underset{q+r=p}{\oplus}\left[H^{q}\left(M_{1}, \mathrm{Z}\right) \otimes H^{r}\left(M_{2}, \mathrm{Z}\right)\right] \\
\oplus \underset{s+t=p+1}{\oplus} \operatorname{Tor}\left[H^{s}\left(M_{1}, Z\right), H^{t}\left(M_{2}, Z\right)\right]
\end{aligned}
$$

Let $\langle q, r\rangle=\operatorname{gcd}\{q, r\}$ denote the greatest common divisor of $q$ and $r$, then we may evaluate (32) using

$$
\begin{gathered}
\mathbf{Z}_{q} \otimes \mathbf{Z}_{r} \cong \mathbf{Z}_{\langle q, r\rangle}, \quad \mathbf{Z} \otimes \mathbf{Z} \cong \mathbf{Z}, \quad \mathbf{Z} \otimes \mathbf{Z}_{p} \cong \mathbf{Z}_{p} \otimes \mathbf{Z} \cong \mathbf{Z}_{p}, \\
\operatorname{Tor}\left[\mathbf{Z}_{q}, \mathbf{Z}_{r}\right] \cong \mathbf{Z}_{\langle q, r\rangle}, \\
\operatorname{Tor}[\mathbf{Z}, \mathbf{Z}]=\operatorname{Tor}\left[\mathbf{Z}_{p}, \mathbf{Z}\right]=\operatorname{Tor}\left[\mathbf{Z}, \mathbf{Z}_{p}\right]=0 .
\end{gathered}
$$

$H^{*}(M, \mathrm{Z})$ is the most general cohomology: one has ${ }^{20}$

Theorem V.4 (Universal Coefficient Theorem): Let $M$ be $a$ manifold and $\mathrm{A}$ be an abelian group. If $\mathrm{A}$ or $H^{*}(M, \mathrm{Z})$ is finitely generated, then

$$
H^{p}(M, \Lambda) \cong H^{p}(M, Z) \otimes \Lambda \oplus \operatorname{Tor}\left[H^{p+1}(M, Z), \mathrm{A}\right]
$$

If $\mathrm{A}$ is torsion free (e.g. $\mathrm{A}=\mathrm{Q}, \mathrm{R}, \mathrm{C}$ ), this yields $H^{p}(M, \mathrm{~A}) \cong H^{P}(M, Z) \otimes \mathrm{A}$, which deletes all finite subgroups of $H^{p}(M, Z)$ and replaces the ones isomorphic to $\mathbb{Z}^{n}$ by $\mathrm{A}^{n}$. Thus for the real- or complex-valued de-Rham cohomology we have

Corollary V.5: Let $M_{1}$ and $M_{2}$ be manifolds and $\mathrm{A}=\mathrm{R}, \mathrm{C}$. If $H^{*}\left(M_{1}, \mathrm{~A}\right)$ or $H^{*}\left(M_{2}, \mathrm{~A}\right)$ is finitely generated then

$$
H^{p}\left(M_{1} \times M_{2}, \mathrm{~A}\right) \cong \underset{q+r=p}{\oplus}\left[H^{q}\left(M_{1}, \mathrm{~A}\right) \otimes H^{r}\left(M_{2}, \mathrm{~A}\right)\right] .
$$

So $H^{*}\left(M_{1} \times M_{2}, \mathrm{~A}\right) \cong H^{*}\left(M_{1}, \mathrm{~A}\right) \otimes H^{*}\left(M_{2}, \mathrm{~A}\right)$, cf. Theorem II.3 and (6). We further state by

Lemma V.6 (Poincaré Lemma, cohomology of $\left.\mathrm{S}^{n}\right):$ For all $n \geqslant 0$ the cohomology of $\mathrm{R}^{n}$ is given

$$
H^{0}\left(\mathbb{R}^{n}, \mathrm{Z}\right) \cong \mathrm{Z} ; \quad H^{p}\left(\mathbb{R}^{n}, \mathrm{Z}\right)=0, \quad \text { for all } p>0 .
$$

For all $n>0$ the cohomology of the $n$-spheres is given by

$$
H^{0}\left(\mathrm{~S}^{n}, \mathrm{Z}\right) \cong \mathrm{Z} ; \quad H^{n}\left(\mathrm{~S}^{n}, \mathrm{Z}\right) \cong \mathbf{Z} ; \quad H^{p}\left(\mathrm{~S}^{n}, \mathrm{Z}\right)=0, \text { for all } p \neq 0, n .
$$

Theorem V.7 (Poincaré duality): If $M$ is a compact and orientable manifold of dimension $n$ (without boundary), then

$$
H^{p}(M, \mathbb{R}) \cong H^{n-p}(M, \mathbb{R})
$$




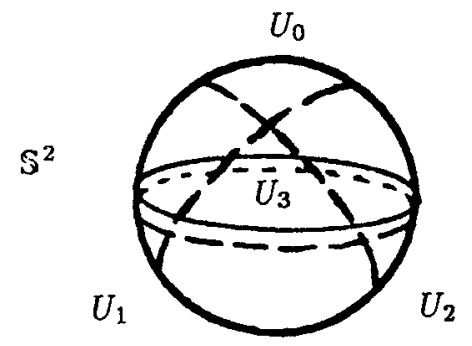

southern hemisphere:

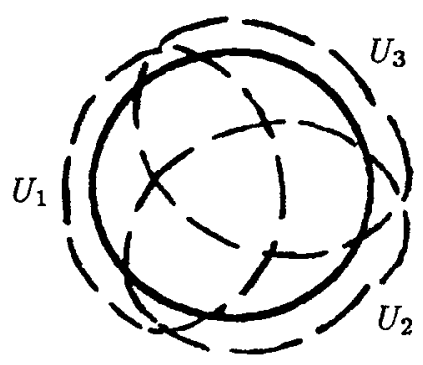

FIG. 1. A good cover on $\mathbf{S}^{2}$.

In our special case of an isolated monopole, where $B_{m}\left(M, \mathrm{SU}_{n}, G_{\mathrm{em}}\right) \cong B_{m}\left(\mathrm{~S}^{2}, \mathrm{SU}_{n}, G_{\mathrm{em}}\right)$ $\times \mathbb{R}_{(t)} \times \mathbf{R}_{(r)}^{+}$, we have $H^{*}\left(B_{m}(M)\right) \cong H^{*}\left(B_{m}\left(\mathbb{S}^{2}\right)\right)$ since for any space $X$ and any contractible space $C$, the spaces $X$ and $X \times C$ are homotopy equivalent and therefore have the same cohomology. (Note that the result for $B_{m}$ also follows from Theorem V.3 and the Poincare lemma.) Now $B_{m}\left(\mathrm{~S}^{2}, \mathrm{SU}_{n}, G_{\mathrm{em}}\right)$ is compact because $\mathrm{S}^{2}$ and $\mathrm{SU}_{n}$ are, which yields Poincare duality for the de-Rham cohomology.

In order to construct $H^{*}(B(M, F, G))$ out of $H^{*}(M)$ and $H^{*}(F)$ one uses the spectral sequence $\left\{E_{n}\right\}_{n \in N_{0}}$ of the Cech-de-Rham complex $C^{* * *}\left(\pi^{-1} \mathfrak{U}, \mathscr{C}\right)$, where $\mathfrak{U}=\left\{U_{\alpha}\right\}_{\alpha \in A}$ is a countable good cover of $M .^{18}$ For a good cover of an $n$-dimensional manifold all finite open intersections $U_{\alpha_{0} \cdots \alpha_{p}}:=U_{\alpha_{0}} \cap \cdots \cap U_{\alpha_{p}}, p \in \mathbf{N}_{0}$ are diffeomorphic to $\mathbb{R}^{n}$. Every paracompact manifold has a good cover $\mathfrak{U}$, and if $M$ is compact, $\mathfrak{U}$ may be chosen to he finite. For our special case, Figure 1 shows a good cover of $\mathrm{S}^{2}$ consisting of four open sets, where $U_{0}$ covers the northern hemisphere and $U_{1} \cup U_{2} \cup U_{3}$ covers the southern hemisphere.

For any countable ordered cover $\mathfrak{U}$ of a manifold $M$ the Čech-de-Rham complex is defined to be the double complex

$$
C^{*, *}(\mathfrak{U}, \mathscr{A}):=\underset{p, q \in \mathrm{N}_{0}}{\oplus} C^{p}\left(\mathfrak{U}, \mathscr{f}_{q}\right)
$$

where

$$
C^{p}\left(\mathfrak{U}, \mathscr{A}_{q}\right):=\prod_{\alpha_{0}<\cdots<\alpha_{p}} \mathscr{A}_{q}\left(U_{\alpha_{0} \cdots \alpha_{p}}\right) .
$$

In every double complex $K^{*, *}:=\oplus_{p, q \in \mathrm{N}_{0}} K^{p, q}$ we have two commuting differential operators: horizontal and vertical homomorphisms $\delta: K^{p, q} \rightarrow K^{p+1, q}$, resp., $d: K^{p, q} \rightarrow K^{p, q+1}$ with $\delta d=d \delta$ and $\delta^{2}=d^{2}=0$. For the Čech-de-Rham complex, $d$ is the exterior differentiation of forms and $\delta$ is the difference operator defined by (" $\wedge$ " denotes omission)

$$
(\delta \omega)_{\alpha_{0} \cdots \alpha_{p+1}}:=\left.\sum_{j=0}^{p+1}(-1)^{j} \omega_{\alpha_{0} \cdots \hat{\alpha}_{j} \cdots \alpha_{p+1}}\right|_{U_{\alpha_{0} \cdots \alpha_{p+1}}},
$$

for all

$$
\omega=\prod_{\alpha_{0}<\cdots<\alpha_{p}} \omega_{\alpha_{0} \cdots \alpha_{p}} \in \prod_{\alpha_{0}<\cdots<\alpha_{p}} \mathscr{A}\left(U_{\alpha_{0} \cdots \alpha_{p}}\right) .
$$




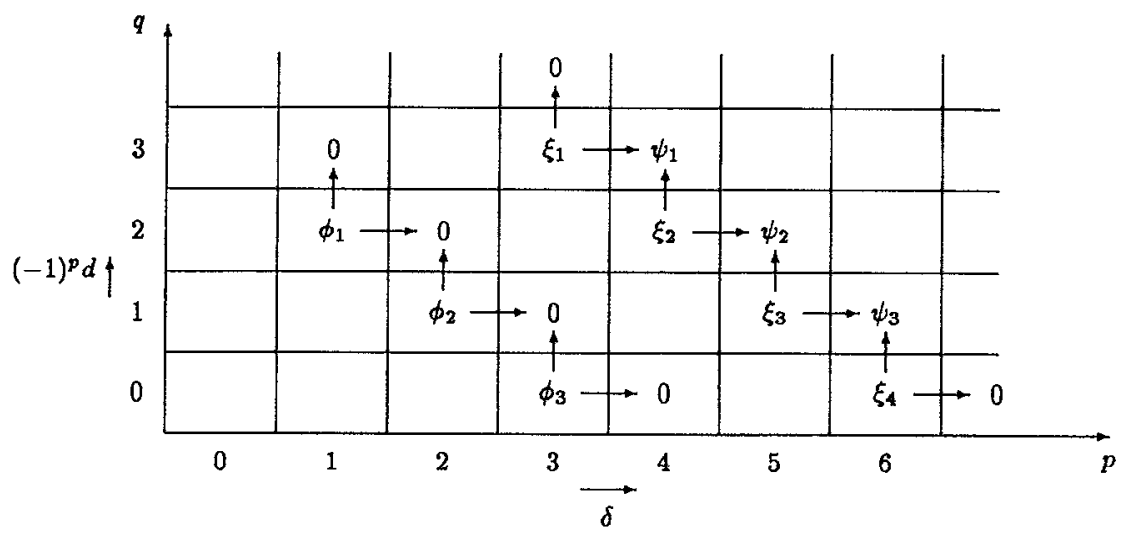

FIG. 2. $D$-closed and $D$-exact elements in a double complex.

Given any double complex $K^{*, *}$, one can form a single (graded) complex $K=$ $\oplus \oplus_{n \in N_{0}} K^{n}$ by summing along the antidiagonal lines, i.e. $K^{n}:=\bigoplus_{p+q=n} K^{p, q}$, and introducing the differential operator $D: K^{n} \rightarrow K^{n+1}$ by:

$$
D:=D^{\prime}+D^{\prime \prime}, \quad D^{\prime}:=\delta, \quad D^{\prime \prime}:=(-1)^{p} d \text { on } K^{p, q} .
$$

The alternation of the sign guarantees $D^{2}=\delta^{2}+\delta d-d \delta+d^{2}=0 . D$ serves for the $D$-cohomology $H_{D}^{*}(K)$ : Figure 2 shows a $D$-closed element $\Phi=\phi_{1}+\phi_{2}+\phi_{3} \subset K$ and a $D$-exact element $\Psi=\psi_{1}+\psi_{2}+\psi_{3}=D \Xi \in K$, where $\Xi=\xi_{1}+\xi_{2}+\xi_{3}+\xi_{4} \in K$ :

$$
\begin{gathered}
D \Phi=0 \Leftrightarrow d \phi_{1}=0, \delta \phi_{1}+d \phi_{2}=0, \\
\delta \phi_{2}-d \phi_{3}=0, \delta \phi_{3}=0, \\
\Psi=D \Xi \Leftrightarrow d \xi_{1}=0, \delta \xi_{1}+d \xi_{2}=\psi_{1}, \\
\delta \xi_{2}-d \xi_{3}=\psi_{2}, \delta \xi_{3}+d \xi_{4}=\psi_{3}, \delta \xi_{4}=0 .
\end{gathered}
$$

Theorem V.8 (Mayer-Vietoris Principle): Let $\mathfrak{U}$ be an open cover of a manifold $M$, then the restriction map $r: \mathscr{A}(M) \rightarrow \Pi_{\alpha} \mathscr{\&}\left(U_{\alpha}\right) \subset C(\mathfrak{U}, \mathscr{C})$ induces an isomorphism:

$$
r^{\star}: H^{*}(M) \rightarrow H_{D}^{*}(C(\mathfrak{U}, \mathscr{\ell})), \quad H^{n}(M) \rightarrow H_{D}^{n}(C(\mathfrak{U}, \mathscr{A})) .
$$

The inverse map that collates together the components of an element in the Čech-de-Rham complex into a global form on $M$ is less intuitive. For any partition of unity $\left\{\rho_{\alpha}\right\}_{\alpha \in A}$ subordinate to $\mathfrak{U}$ define $K: C^{p}\left(\mathfrak{U}, \mathscr{C}_{q}\right) \rightarrow C^{p-1}\left(\mathfrak{U}, \mathscr{A}_{q}\right): \omega \mapsto K \omega$ by

$$
(K \omega)_{\alpha_{0} \cdots \alpha_{p-1}}:=\sum_{\alpha \in A} \rho_{\alpha} \omega_{\alpha \alpha_{0} \cdots \alpha_{p-1}}
$$

where we allow indices in any order, even with repetitions, subject to the convention that the forms are totally antisymmetric under permutation of the indices. We have $K \delta+\delta K=\mathrm{id}$ and the following theorem holds: ${ }^{18}$ 


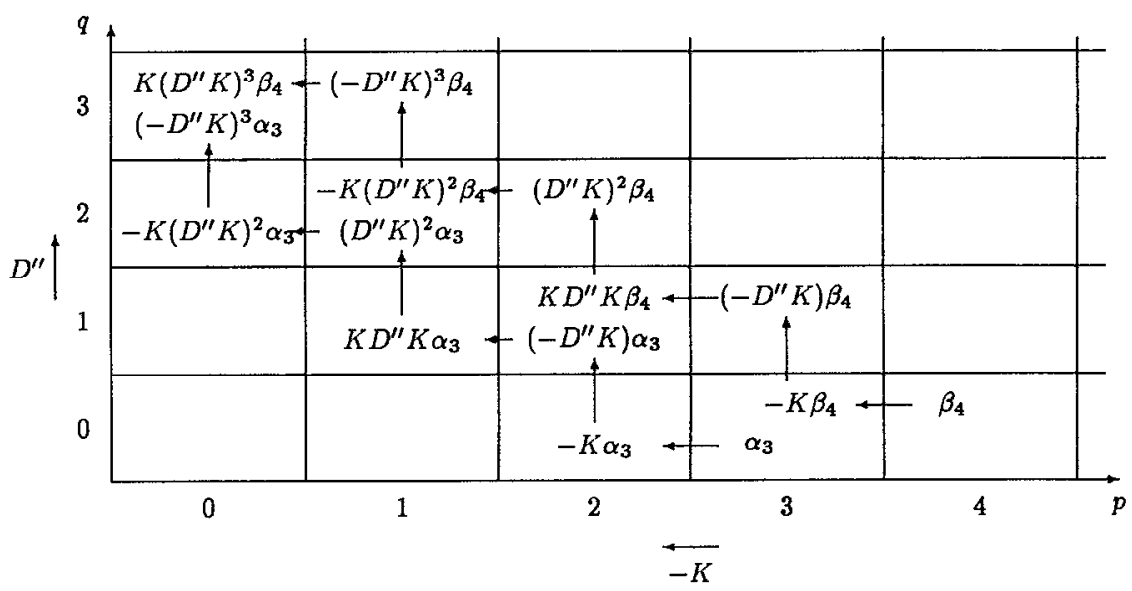

FIG. 3. Illustration of the Collating formula.

Theorem V.9 (Collating Formula): In the Čech-de-Rham complex, let $\alpha=\sum_{i=0}^{n} \alpha_{i} \in C\left(\mathfrak{U}, \mathscr{A}^{n}\right)^{n}$ with $\alpha_{i} \in C^{i}\left(\mathfrak{U}, \mathscr{A}_{n-i}\right)$ and $D \alpha=\beta=\Sigma_{i=0}^{n+1} \beta_{i}$ with $\beta_{i}$ $\in C^{i}\left(\mathfrak{U}, \mathcal{B}_{n+1-i}\right)$, and define $K$ by (33). Then

$$
f(\alpha):=\sum_{i=0}^{n}\left(-D^{\prime \prime} K\right)^{i} \alpha_{i}-\sum_{i=0}^{n} K\left(-D^{\prime \prime} K\right)^{i} \beta_{i+1}
$$

$\in C^{0}\left(\mathfrak{U}, \mathscr{A}_{n}\right)$ is a global form on $M$ (resp., the restriction of such a form to the sets $U_{\alpha}$ in the cover $\mathfrak{U})$. The function $f: C(\mathfrak{U}, \mathscr{h}) \rightarrow \mathscr{L}(M)$ is left inverse to $r$, so $f \circ r=\mathrm{id},(M)$ while

$$
r \circ f: C(\mathfrak{U}, \mathscr{A}) \rightarrow C(\mathfrak{U}, \mathscr{t}), \quad C(\mathfrak{U}, \mathscr{b})^{n} \rightarrow C^{0}\left(\mathfrak{U}, \mathscr{A}_{n}\right)
$$

associates with every $D$-closed element in $C(\mathfrak{U}, \mathscr{A})^{n}$ an element in the same cohomology class of $H_{D}^{n}(C(\mathfrak{U}, \hat{\ell}))$. Thus the induced maps $f^{\star}$ and $r^{\star}$ on the cohomology level are inverse isomorphisms.

Figure 3 illustrates how the components $\alpha_{3}$ and $\beta_{4}$ of $\alpha$ and $\beta$ are mapped onto elements in $C^{0}\left(\mathcal{U}, A_{3}\right)$. For a global form on $M$, all components $\alpha_{i}$ and $\beta_{i}$ must be mapped like this.

A filtered complex $K$ is a sequence $K=K_{0} \supseteq K_{1} \supseteq \cdots$, where the $K_{i}$ are subcomplexes of $K$ with $D K_{i} \subseteq K_{i}$. For every filtered complex $K$, we have an associated graded complex $G K$ : $=\oplus_{p=0}^{\infty} K_{p} / K_{p+1}$. For a double complex the sequence $K_{p}:=\oplus_{i=p}^{\infty} \oplus_{q=0}^{\infty} K^{i, q}, p \in \mathbf{N}_{0}$ is a filtration by the columns of $K$ with associated graded complex

$$
G K=\underset{p=0}{\oplus} K_{p} / K_{p+1}=\underset{p=0}{\oplus} u\left[\left(\underset{q=0}{\oplus} K^{p, q}\right)+K_{p+1}\right]
$$

Obviously the induced differential operator on $G K$ is just $(-1)^{p} d$.

By a spectral sequence we mean a sequence of complexes $\left\{E_{r}\right\}_{r \in \mathrm{N}_{0}}$ with differential operators $D_{r}$, where every $E_{r}$ is the cohomology of its predecessor: $E_{r+1}=H_{D_{r}}^{*}\left(E_{r}\right)$. If $E_{R}$ becomes stationary, i.e. $E_{r}-E_{r+1}$ for all $r \geqslant R$, we denote $E_{R}$ by $E_{\infty}$ and say that the spectral sequence converges to some filtered complex $H$ if $E_{\infty} \cong G H$. We get the spectral sequence of a double complex by setting $E_{0}=G K$ from (34) and defining $D_{r}$ to be the differential operator induced by $D$ on $E_{r}$, so $E_{r+1}=H_{D}^{*}\left(E_{r}\right)$. We say that an element $\beta \in K^{*, *}$ lives to $E_{r}, r>0$ if it represents 


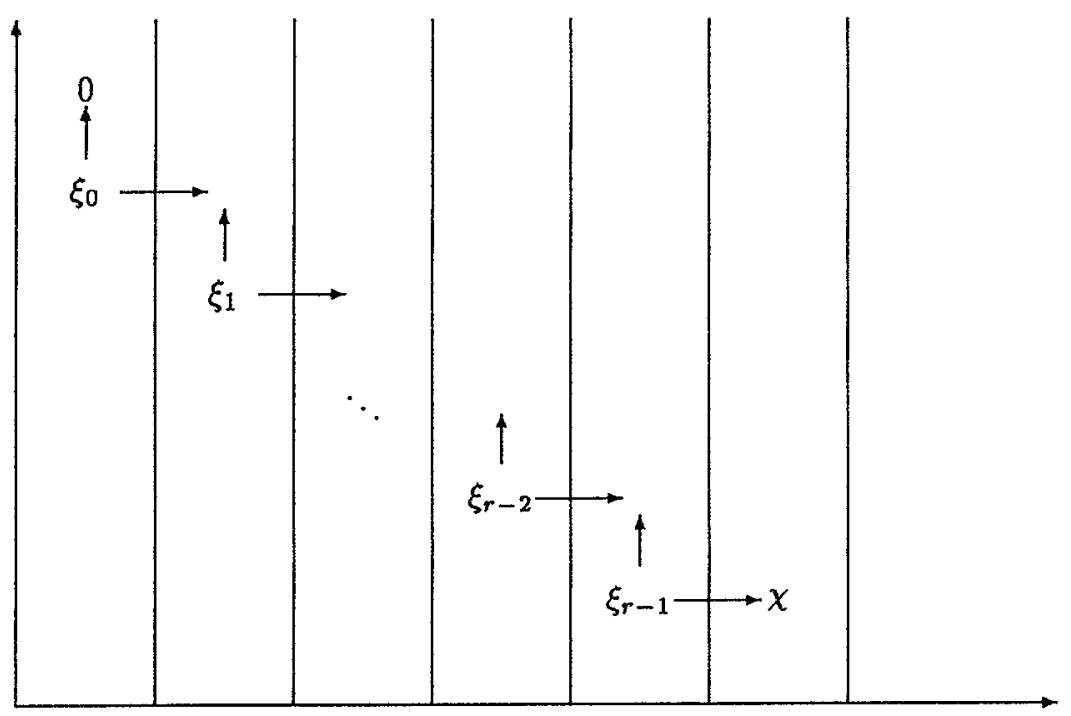

FIG. 4. Illustration of the differential operator $D_{r}: D_{r}\left[\xi_{0}\right]_{r}=\left[\delta \xi_{r-1}\right]_{r}=[\chi]_{r}$.

a cohomology class $[\beta]_{r} \in E_{r}$, resp. equivalently, if $\beta$ is $D$-closed in $E_{0}, E_{1}, \ldots, E_{r-1}$ : $D_{i}[\beta]_{i}=0, i=0, \ldots, r-1$. Then $\beta$ is $d$-closed and we have a "zigzag" $\Xi=\xi_{0}+\xi_{1}+\ldots+\xi_{r-1}$ of elements $\xi_{i} \in K^{* * *}$ with $\xi_{0}:=\beta$ and (cf. Figure 4)

$$
D^{\prime} \xi_{i}=\delta \xi_{i}=-D^{\prime \prime} \xi_{i+1}, \quad i=0, \ldots, r-2 .
$$

Now $D_{r}[\beta]_{r}=\left[\delta \xi_{r-1}\right]_{r}=[\chi]_{r}$, so $D_{r}$ is given by $\delta$ at the end of the zig-zag.

Thus like $K^{*, *}$ every $E_{r}$ is a double complex, too: $E_{r}=\oplus_{p, q \geqslant 0} E_{r}^{p, q}$ and $D_{r}$ shifts the bidegrees by $(r,-r+1): D_{r}: E_{r}^{p, q} \rightarrow E_{r}^{p+r, q-r+1}$. Obviously

$$
E_{1}=H_{d}(K) \text { and } E_{2}=H_{\delta}\left(H_{d}(K)\right) \text {. }
$$

One can prove ${ }^{18}$ that this spectral sequence converges to the $D$-cohomology of $K$ :

$$
G H_{D}^{n}(K)=\underset{p+q=n}{\bigoplus} E_{\infty}^{p, q}(K)
$$

and thus for the Čech-de-Rham complex the Mayer-Vietoris principle proves convergence to the de-Rham cohomology of $M$.

For a bundle $B(M, F)$, if $\mathfrak{U}$ is a good cover of $M, \pi^{-1} \mathfrak{U}$ is a cover of $B$ and for all $U_{\alpha_{0} \cdots \alpha_{p}}$ we have $\pi^{-1}\left(U_{\alpha_{0} \cdots \alpha_{p}}\right) \cong \mathbf{R}^{n} \times F$, so $H^{q}\left(\pi^{-1}\left(U_{\alpha_{0} \cdots \alpha_{p}}\right), Z\right) \cong H^{q}(F, Z)$ by Lemma V.6. Corresponding to (36) and (37) we have: ${ }^{18,20}$

Theorem V.10 (Leray's Theorem): If $B(M, F)$ is a fiber bundle and $\mathfrak{U}=\left\{U_{\alpha}\right\}_{\alpha \in A}$ is a good cover of $M$ then there is a spectral sequence converging to $H^{*}(B, \mathbb{Z})$ with $E_{1}$ term

$$
E_{1}^{p, q}=\prod_{\alpha_{0}<\cdots<\alpha_{p}} H^{q}\left(\pi^{-1}\left(U_{\alpha_{0} \cdots \alpha_{p}}\right), Z\right) \cong \prod_{\alpha_{0}<\cdots<\alpha_{p}} H^{q}(F, Z)
$$


If $H^{*}(F, Z)$ is finitely generated and in addition $M$ simply connected or $B \cong M \times F$, then $E_{2}^{p, q}=H^{p}\left(M, H^{q}(F, Z)\right)$

$$
\cong H^{p}(M, \mathrm{Z}) \otimes H^{q}(F, \mathrm{Z}) \oplus \operatorname{Tor}\left[H^{p+1}(M, \mathrm{Z}), H^{q}(F, \mathrm{Z})\right] .
$$

Leray's theorem gives a proof for the Künneth formula, because all forms in $E_{2}$ are closed global forms on $M \times F$ for which $d=\delta=0$ and thus $D_{2}=0$. So $E_{2}$ becomes stationary, which proves Theorem V.3. ( $\operatorname{Tor}\left[H^{0}(M, Z), H^{q}(F, Z)\right]=0$, since $H^{0}(M, Z)$ is free.)

Finally we give the notion of transgressive elements: for a connected fiber $F$ an element $\omega \in H^{q}(F) E_{1}^{0, q}$ is called transgressive iff it lives to $E_{q+1}$. These represent forms on the bundle because of the following theorem: ${ }^{18}$

Theorem V. 11: Let $B(M, F)$ be a bundle with connected fiber $F$. Then $\omega \in H^{q}(F)$ is transgressive iff it is the restriction of a $\psi \in \mathscr{H}_{q}(B)$ with $d \psi=\pi^{\star} \tau$ for some $\tau \in \mathscr{G}_{q+1}(M)$.

We find $\psi$ and $\tau$ using the Collating formula: first we take a zigzag $\exists=\xi_{0}+\ldots+\xi_{q}$ according to (35) and Figure 4 (where $q=r-1$ ). Now $d \chi=d \delta \xi_{q}=\delta d \xi_{q}= \pm \delta \delta \xi_{q-1}=0$, thus the components of $\chi$ are locally constant on $\pi^{-1}\left(U_{\alpha_{0} \cdots \alpha_{p}}\right) \cong U_{\alpha_{0} \cdots \alpha_{p}} \times F$, and because $F$ is connected we have $\chi=\pi^{\star} \beta$ with $\beta \in C^{q+1}\left(\mathfrak{U}, \mathscr{C}_{0}\right)$. Now Theorem V.9 yields

$$
\psi:=f(\Xi)=\sum_{i=0}^{q}\left(-D^{\prime \prime} K\right)^{i} \xi_{i}-K\left(-D^{\prime \prime} K\right)^{q} \pi^{\star} \beta \in \mathscr{A}_{q}(B)
$$

and

$$
d \psi=\left(-D^{\prime \prime} K\right)^{q+1} \pi^{\star} \beta=\pi^{\star} \tau \in \mathscr{A}_{q+1}(B),
$$

where

$$
\tau=\left(-D^{\prime \prime} K\right)^{q+1} \beta \in \mathscr{H}_{q+1}(M) .
$$

Now closed forms on $F$ transform into closed forms on $B$ as follows: $[\chi]_{q+1}=0 \Leftrightarrow \exists \Xi \Xi^{\prime}=\xi_{0}+\xi_{1}^{\prime}+\ldots+\xi_{q}^{\prime}: \delta \xi_{q}^{\prime}=0 \Leftrightarrow \exists \psi=f\left(\Xi^{\prime}\right): d \psi=0$. Thus we get

Corollary V.12: For any $\omega \in H^{q}(F)$, there exists $\psi \in H^{q}(B)$ such that $\omega$ is the restriction of $\psi$ iff $\omega$ not only is transgressive but even lives to $E_{q+2}$.

\section{Spectral sequences of the skyrmion bundle}

Before applying these results to the skyrmion bundle in generality let us first look on our special case where $H^{*}\left(B_{m}\left(M, \mathrm{SU}_{n}, G_{\mathrm{em}}\right)\right) \cong H^{*}\left(B_{m}\left(\mathrm{~S}^{2}, \mathrm{SU}_{n}, G_{\mathrm{em}}\right)\right)$. Since $H^{*}\left(\mathrm{~S}^{2}\right)$ is torsion free, Leray's theorem yields:

$$
E_{2}^{p, q}=H^{p}\left(\mathrm{~S}^{2}, \mathrm{Z}\right) \otimes H^{q}\left(\mathrm{SU}_{n}, \mathrm{Z}\right)= \begin{cases}H^{q}\left(\mathrm{SU}_{n}, \mathrm{Z}\right), & \text { for } p=0,2 \\ 0, & \text { otherwise }\end{cases}
$$

Figure 5 shows $E_{2}$ for $n=2,3,4$. For $n=2,3$ obviously $D_{2}^{p, q}: E_{2}^{p, q} \rightarrow E_{2}^{p+2, q-1}$ is the zero map, and so is $D_{i}$ for all $i \geqslant 2$. Thus $E_{2}$ becomes stationary and we have

$$
H^{k}\left(B_{m}\left(M, \mathrm{SU}_{n}, G_{\mathrm{em}}\right), \mathrm{Z}\right) \cong H^{k}\left(M \times \mathrm{SU}_{n}, \mathrm{Z}\right)=\underset{p+q=k}{\oplus} H^{p}\left(\mathrm{~S}^{2}, \mathrm{Z}\right) \otimes H^{q}\left(\mathrm{SU}_{n}, Z\right), \quad n=2,3 .
$$

In the diagram for $n=4$ the morphism $D_{2}^{0,8}: Z \rightarrow Z$ is given by $D_{2}^{0,8}(m)=k \cdot m$ for an integer $k \in Z$. In particular, it is either 0 or injective. If $k \neq 0$, then $E_{3}^{0,8}=\{0\}=E_{\infty}^{0,8}$, while $E_{3}^{2,7} \cong \mathrm{Z}_{|k|} \cong E_{\infty}^{2,7}$. So for our deciding whether $D_{2}=0$, we must inspect the induced differential operators more closely, and we will do this in the general case. 

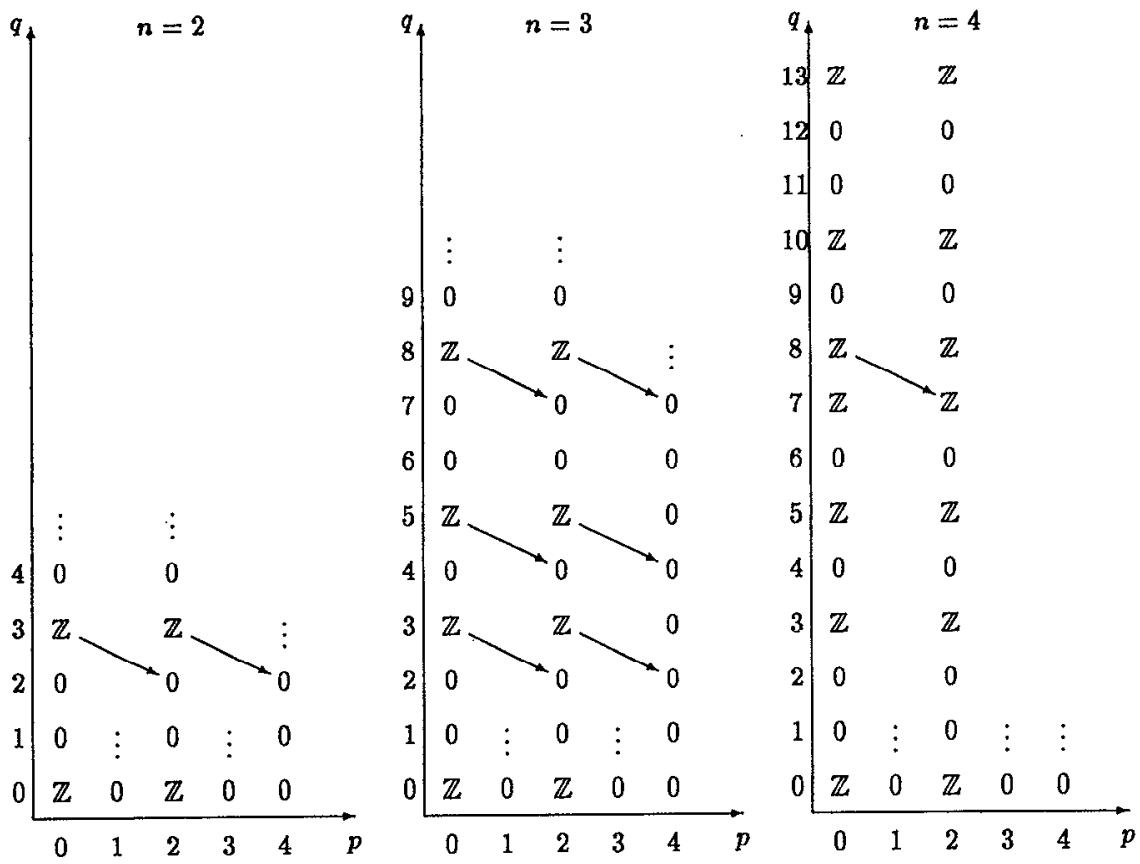

FIG. 5. $E_{2}$ term of the spectral sequence for the skyrmion bundle.

Let us compute the zigzag for $\omega_{2 l+1}$ in a skyrmion bundle, where the bundle atlas is given over a good cover $\mathfrak{U}$. Using the local trivializations we inject $\omega_{2 l+1}$ into $C^{0}\left(\pi^{-1} \mathfrak{U}, \mathscr{A}_{2 l+1}\right)$, so $\xi_{0}$ in Figure 4 is given by $\left(\xi_{0}\right)_{\alpha}=\omega_{2 l+1}^{\alpha}$. For $U_{\alpha \beta} \neq \varnothing$ by Lemma IV.2 and (3),

$$
\begin{aligned}
\left(\delta \xi_{0}\right)_{\alpha \beta}=\left.\left(\omega_{2 l+1}^{\beta}-\omega_{2 l+1}^{\alpha}\right)\right|_{U_{\alpha \beta}} & =(2 l+1) \text { ie } d g_{\alpha \beta} \wedge\left(\rho_{2 l}^{Q}-\lambda_{2 l}^{Q}\right), \\
& =d\left[(2 l+1) \text { ie } d g_{\beta \alpha} \wedge\left(\rho_{2 l-1}^{Q}+\lambda_{2 l-1}^{Q}\right)^{\alpha / \beta}\right]=\left(d \xi_{1}\right)_{\alpha \beta},
\end{aligned}
$$

where

$$
\begin{aligned}
\left(\xi_{1}\right)_{\alpha \beta}: & =\text { ie } d g_{\beta \alpha} \wedge\left(\chi_{2 l-1}^{1}\right)^{\alpha / \beta} \\
: & =(2 l+1) \text { ie } d g_{\beta \alpha} \wedge\left(\rho \frac{Q}{2 l-1}+\lambda_{2 l-1}^{Q}\right)^{\alpha / \beta} .
\end{aligned}
$$

Using Lemma IV.2 again and $d g_{\gamma \alpha}=d g_{\gamma \beta}+d g_{\beta \alpha}$ on $U_{\alpha \beta \gamma} \neq \varnothing$ we have

$$
\begin{aligned}
\left(\delta \xi_{1}\right)_{\alpha \beta \gamma}=\left.\left[\left(\xi_{1}\right)_{\beta \gamma}-\left(\xi_{1}\right)_{\alpha \gamma}+\left(\xi_{1}\right)_{\alpha \beta}\right]\right|_{U_{\alpha \beta \gamma}}= & \text { ie } d g_{\gamma \beta} \wedge\left[\left(\chi_{2 l-1}^{1}\right)^{\beta}-\left(\chi_{2 l-1}^{1}\right)^{\alpha}\right] \\
= & (2 l+1)(i e)^{2} d g_{\gamma \beta} \wedge d g_{\alpha \beta} \\
& \wedge\left[\sum_{j=0}^{l-1} \operatorname{Tr}\left(Q R^{2 j} Q R^{2 l-2 j-2}-Q L^{2 j} Q L^{2 l-2 j-2}\right)\right. \\
& \left.+2 \sum_{j=1}^{l-1} \operatorname{Tr}\left(Q U L^{2 j-1} Q L^{2 l-2 j-1} U^{\dagger}\right)\right]^{\alpha / \beta / \gamma} \\
& =\left(-d \xi_{2}\right)_{\alpha \beta \gamma}
\end{aligned}
$$




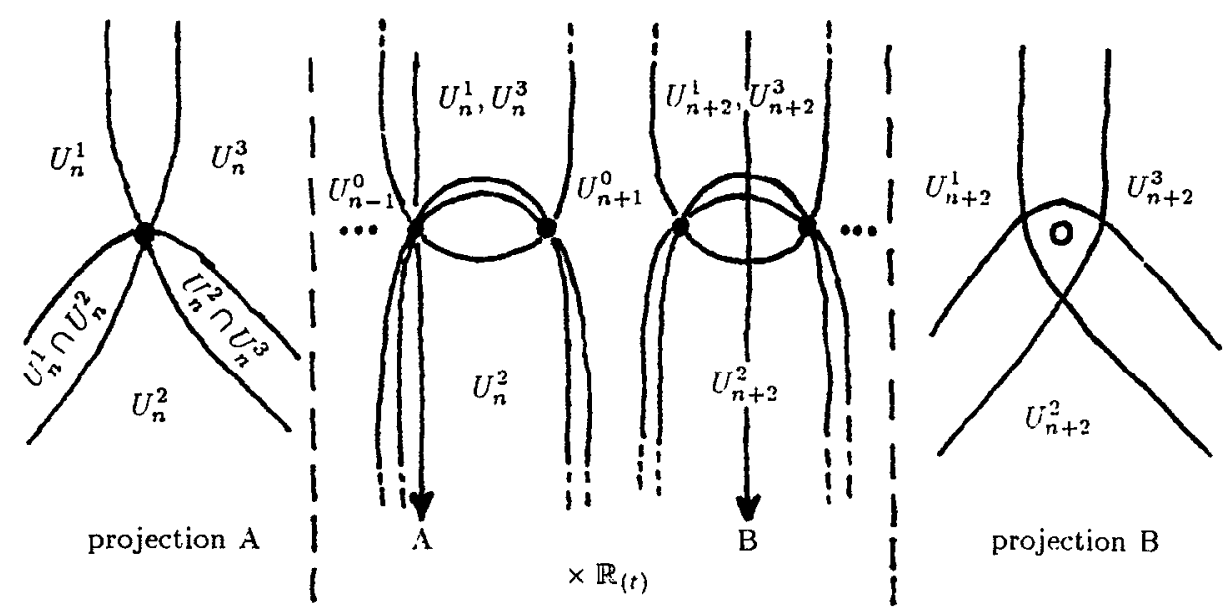

FIG. 6. A good cover for a chain of monopoles.

with

$$
\left(\xi_{2}\right)_{\alpha \beta \gamma}=(i e)^{2} d g_{\gamma \beta} \wedge d g_{\beta \alpha} \wedge\left(\chi_{2 l-3}^{2}\right)^{\alpha / \beta / \gamma} .
$$

For $\chi_{2 l-3}^{2} \in \mathscr{H}_{2 l-3}\left(\mathrm{SU}_{n}\right)$ we may take using (3) and (4)

$$
\begin{aligned}
\chi_{2 l-3}^{2}=(2 l+1)\left[2\left(\rho_{2 l-3}^{Q^{2}}+\lambda \frac{Q^{2}}{2 l-3}\right)+\sum_{j=1}^{l-2} \operatorname{Tr}\left(Q R^{2 j-1} Q R^{2 l-2 j-2}+Q L^{2 j-1} Q L^{2 l-2 j-2}\right)\right. \\
\left.+\sum_{j=1}^{l-1} \operatorname{Tr}\left(Q U L^{2 j-1} Q L^{2 l-2 j-2} U^{\dagger}+Q U L^{2 j-2} Q L^{2 l-2 j-1} U^{\dagger}\right)\right] .
\end{aligned}
$$

If we define $\nu_{2 l-4} \in \mathcal{B}_{2 l-4}\left(\mathrm{SU}_{n}\right)$ by $\left(\chi_{2 l-3}^{2}\right)^{\alpha}=\left(\chi_{2 l-3}^{2}\right)^{\beta}+i e d g_{\alpha \beta} \wedge \nu_{2 l-4}^{\alpha / \beta}$ we get

$$
\begin{aligned}
\left(\delta \xi_{2}\right)_{\alpha \beta \gamma \delta} & =\left.\left[\left(\xi_{2}\right)_{\beta \gamma \delta}-\left(\xi_{2}\right)_{\alpha \gamma \delta}+\left(\xi_{2}\right)_{\alpha \beta \delta}-\left(\xi_{2}\right)_{\alpha \beta \gamma}\right]\right|_{U_{\alpha \beta \gamma \delta}} \\
& =(2 l+1)(i e)^{3} d g_{\delta \gamma} \wedge d g_{\gamma \beta} \wedge d g_{\beta \alpha} \wedge \nu_{2 l-4}^{\alpha / \beta l \gamma / \delta}
\end{aligned}
$$

in the next step. Let us terminate at this point. $D_{2}\left[\omega_{2 l+1}\right]_{2}=\left[\delta \xi_{1}\right]_{2}$, so whenever $\delta \xi_{1}=0$, $\omega_{2 n-1}$ lives to $E_{\infty}$. In any event this is the case if for our cover $d g_{\gamma \beta} \wedge d g_{\beta \alpha}=0$ for all combinations of $\alpha, \beta$ and $\gamma$. For example, the cover in Figure 1 obeys this condition, but also the cover in Figure 6, if we examine a whole chain of monopole locations. At any time any distribution of countable magnetic monopoles at isolated locations can be continuously deformed into such a chain.

Moreover, if all $d g_{\gamma \beta} \wedge d g_{\beta \alpha}=0$ then $D_{2}=0$ everywhere, because for $\left(\tilde{\xi}_{0}\right)_{\alpha}=\omega_{l_{1}}^{u} \wedge \cdots \wedge \omega_{l_{k}}^{\alpha}$ with $l_{j}>1$ odd, we get by Lemma IV.2

$$
\left(\tilde{\xi}_{1}\right)_{\alpha \beta}=\sum_{j=1}^{k} l_{j} i e d g_{\beta \alpha} \wedge \omega_{l_{1}}^{\alpha / \beta} \wedge \cdots \wedge \omega_{l_{j-1}}^{\alpha / \beta} \wedge\left(\rho_{l_{j}-2}^{Q}+\lambda l_{l_{j}-2}^{Q}\right)^{\alpha / \beta} \wedge \omega_{l_{j+1}}^{\alpha / \beta} \wedge \cdots \wedge \omega_{l_{k}}^{\alpha / \beta},
$$

and thus $\delta_{1} \tilde{\xi}_{1}=0$. We have found: 
Theorem V.13: The cohomology of the skyrmion bundle $B_{m}\left(M, \mathrm{SU}_{n}, G_{e m}\right)$ is independent of the monopole charge $m g_{D}$. It is isomorphic to the cohomology of $M \times \mathrm{SU}_{n}$ : for all $k \in \mathrm{N}_{0}$,

$$
H^{k}\left(B_{m}\left(M, \mathrm{SU}_{n}, G_{e m}\right), \mathrm{Z}\right) \cong \underset{p+q=k}{\oplus} H^{p}(M, \mathrm{Z}) \otimes H^{q}\left(\mathrm{SU}_{n}, \mathrm{Z}\right)
$$

The same holds for all skyrmion bundles of manifolds, where a good cover $\mathfrak{U}=\left\{U_{\alpha}\right\}_{\alpha \in A}$ of $M$ exists such that $d g_{\gamma \beta} \wedge d g_{\beta \alpha}=0$ for all $\alpha, \beta, \gamma \in A$.

Table III lists $H^{p}\left(B_{m}\left(M, \mathrm{SU}_{n}, G_{\mathrm{em}}\right), \mathrm{Z}\right)$ for $n=N_{F}=2, \ldots, 6$ and $p \geqslant 38$ using (6), Lemma V.6 and the Künneth formula. Finally, since $\chi_{1}^{1}=\rho_{1}^{Q}+\lambda_{1}^{Q}$ and $\chi_{1}^{2}=10\left(\rho_{1}^{Q^{2}}+\lambda_{1}^{Q^{2}}\right)$ $+5 \operatorname{Tr}\left(Q d U Q U^{\dagger}-Q U Q d U^{\dagger}\right)$ are global forms, we have proved that $D_{2}\left(\omega_{3}\right)=D_{3}\left(\omega_{5}\right)=0$ for every skyrmion bundle, so $\omega_{3}$ and $\omega_{5}$ live to $E_{\infty}$ and we have

$$
H^{3}\left(B\left(M, \mathrm{SU}_{n}, G_{\mathrm{em}}\right), \mathrm{Z}\right) \cong \mathrm{Z} \text { and } H^{5}\left(B\left(M, \mathrm{SU}_{n}, G_{\mathrm{em}}\right), \mathrm{Z}\right) \cong \mathrm{Z} .
$$

Theorem V.9 exhibits representatives for the cohomology groups. For the single monopole, using the trivializations $\psi^{(+)}$over $U_{0}$, resp., $\psi^{(-)}$over $U_{\alpha}, \alpha=1,2,3$ and $\rho_{+}:=\rho_{0}$, resp., $\rho_{-}:=\Sigma_{\alpha=1}^{3} \rho_{\alpha}$ as a partition of unity, we get for $q=l_{1}+\cdots+l_{k}$

$$
\begin{aligned}
\left(\omega_{q}^{m}\right)^{(+)}= & \omega_{l_{1}}^{(+)} \wedge \cdots \wedge \omega_{l_{k}}^{(+)} \\
& +d\left\{\rho_{-}\left[\sum_{j=1}^{k} l_{j} i e d g-{ }^{\prime} \wedge \omega_{l_{1}}^{(+)} \wedge \cdots \wedge\left(\rho_{l_{j}-2}^{Q}+\lambda_{l_{j}-2}^{Q}\right)^{(+)} \wedge \cdots \wedge \omega_{l_{k}}^{(+)}\right]\right\}, \\
\left(\omega_{q}^{m}\right)^{(-)}= & \omega_{l_{1}}^{(-)} \wedge \cdots \wedge \omega_{l_{k}}^{(-)} \\
& -d\left\{\rho_{+}\left[\sum_{j=1}^{k} l_{j} i e d g_{-+} \wedge \omega_{l_{1}}^{(-)} \wedge \cdots \wedge\left(\rho_{l_{j}-2}^{Q}+\lambda{ }_{l_{j}-2}^{Q}\right)^{(-)} \wedge \cdots \wedge \omega_{l_{k}}^{(-)}\right]\right\}
\end{aligned}
$$

as global $q$-forms generating $H^{q}\left(B_{m}\left(M, \mathrm{SU}_{n}, G_{\mathrm{em}}\right)\right)$. We can even take-cf. (10)-

$$
\rho_{+} d g_{-+}:=\frac{1}{2}(1+\cos \theta) d g_{-+}=-A_{\mathrm{M}}^{(-)}, \quad \rho_{-} d g_{-+}:=\frac{1}{2}(1-\cos \theta) d g_{-+}=+A_{\mathrm{M}}^{(+)} ;
$$

so all

$$
\left(\omega_{q}^{m}\right)^{( \pm)}=\omega_{l_{1}}^{( \pm)} \wedge \ldots \wedge \omega_{l_{k}}^{( \pm)}+\dot{d}\left\{i e A_{\mathrm{M}}^{( \pm)} \wedge\left[\sum_{j=1}^{k} l_{j} \omega_{l_{1}}^{( \pm)} \wedge \ldots \wedge\left(\rho_{l_{j}-2}^{Q}+\lambda l_{l_{j}-2}\right)^{( \pm)} \wedge \ldots \wedge \omega_{l_{k}}^{( \pm)}\right]\right\}
$$

are global forms, which are gauge invariant: $L_{g}^{\star}\left(\omega_{q}^{m}\right)=\omega_{q}^{m}$ for all $g \in G_{\mathrm{em}}$ [cf. (11)] andtogether with $\pi^{\star} \omega_{2}\left(\mathrm{~S}^{2}\right) \wedge \omega_{q}^{m}\left[\right.$ where $\omega_{2}\left(\mathrm{~S}^{2}\right)$ is the volume form on $\left.\mathrm{S}^{2}\right]$-generate the cohomology of $B_{m}\left(M, \mathrm{SU}_{n}, G_{\mathrm{em}}\right)$.

\section{Cohomology and connection on $B\left(M, \mathrm{SU}_{n}, G_{\mathrm{em}}\right)$}

Because the connection $\Gamma$ on $B$ defines an additional structure, we could not expect to get forms that are adapted to $\Gamma$. The form $\left(\omega_{q}^{m}\right)^{( \pm)}$only contains the gauge potential of the monopole which is closely related to the structure of $P$. In general we cannot substitute $A_{\mathrm{M}}^{( \pm)}$by $A^{( \pm)}$ because $\left(A_{\mathrm{M}}^{( \pm)}-A^{( \pm)}\right) \wedge\left[\Sigma_{j=1}^{k} l_{j} \omega_{l_{1}}^{( \pm)} \wedge \ldots \wedge\left(\rho_{l_{j}-2}^{Q}+\lambda l_{l_{j}-2}^{Q}\right)^{( \pm)} \wedge \ldots \wedge \omega_{l_{k}}^{( \pm)}\right]$is not global; it is global only for $\omega_{3}$. Nevertheless we have the following lemma:

Lemma V.14: Let $\mathfrak{U}=\left\{U_{\alpha}\right\}_{\alpha \in A}$ be a cover of $M,\left\{A^{\alpha} \in \mathscr{B}_{1}\left(U_{\alpha}, \mathfrak{g}\right)\right\}_{\alpha \in A}$ a family such that (17) holds and $\left\{\rho_{\alpha}\right\}_{\alpha \in A}$ a partition of unity subordinate to $\mathfrak{U}$, then $\left\{A^{\alpha}-\Sigma_{\gamma \in A} \rho_{\gamma} d g_{\gamma \alpha}\right\}_{\alpha \in A}$ defines a global $\mathbf{g}$-valued 1-form on $M$. 
TABLE III. Cohomology of the skyrmion bundle $H^{\rho}\left(B_{m}\left(M, \mathrm{SU}_{n}, G_{\mathrm{em}}\right), Z\right)$.

\begin{tabular}{|c|c|c|c|c|c|c|c|c|c|c|c|c|c|c|}
\hline$n$ & $p=0$ & 1 & 2 & 3 & 4 & 5 & 6 & 7 & 8 & 9 & 10 & 11 & 12 & 13 \\
\hline 2 & $Z$ & 0 & $\mathbf{Z}$ & $\mathrm{Z}$ & 0 & $\mathbf{Z}$ & 0 & 0 & 0 & 0 & 0 & 0 & 0 & 0 \\
\hline 3 & $\mathrm{Z}$ & 0 & $\mathbf{Z}$ & $\mathrm{Z}$ & 0 & $Z^{2}$ & 0 & $\mathbf{Z}$ & $\mathbf{Z}$ & 0 & $\mathbf{Z}$ & 0 & 0 & 0 \\
\hline 4 & $\mathrm{Z}$ & 0 & $\mathrm{Z}$ & $z$ & 0 & $Z^{2}$ & 0 & $\mathbf{Z}^{2}$ & $\mathrm{Z}$ & $\mathrm{Z}$ & $\mathbf{Z}^{2}$ & 0 & $\mathrm{Z}^{2}$ & 0 \\
\hline 5 & $Z$ & 0 & $\mathrm{Z}$ & $\bar{Z}$ & 0 & $Z^{2}$ & 0 & $Z^{2}$ & $\mathbf{Z}$ & $\mathbf{Z}^{2}$ & $\mathrm{Z}^{2}$ & $\mathrm{Z}$ & $Z^{3}$ & 0 \\
\hline 6 & $\mathbf{Z}$ & 0 & $\mathrm{Z}$ & $\mathbf{Z}$ & 0 & $z^{2}$ & 0 & $Z^{2}$ & $Z$ & $\mathbf{Z}^{2}$ & $Z^{2}$ & $Z^{2}$ & $Z^{3}$ & $Z$ \\
\hline$n$ & $p=14$ & 15 & 16 & 17 & 18 & 19 & 20 & 21 & 22 & 23 & 24 & 25 & 26 & 27 \\
\hline 2 & 0 & 0 & 0 & 0 & 0 & 0 & 0 & 0 & 0 & 0 & 0 & 0 & 0 & 0 \\
\hline 3 & 0 & 0 & 0 & 0 & 0 & 0 & 0 & 0 & 0 & 0 & 0 & 0 & 0 & 0 \\
\hline 4 & $\mathbf{Z}$ & $\mathbf{Z}$ & 0 & $\mathbf{Z}$ & 0 & 0 & 0 & 0 & 0 & 0 & 0 & 0 & 0 & 0 \\
\hline 5 & $\mathrm{Z}^{3}$ & $\mathrm{Z}$ & $Z^{2}$ & $Z^{2}$ & $\mathrm{Z}$ & $\mathbf{Z}^{2}$ & 0 & $\mathbf{Z}^{2}$ & 0 & Z & $Z$ & 0 & $\mathrm{Z}$ & 0 \\
\hline 6 & $Z^{4}$ & $\mathrm{Z}$ & $Z^{4}$ & $\mathrm{Z}^{2}$ & $\mathrm{Z}^{3}$ & $Z^{3}$ & $Z^{2}$ & $Z^{4}$ & $\mathrm{Z}$ & $Z^{4}$ & $\mathbf{Z}$ & $z^{3}$ & $Z^{2}$ & $\mathrm{Z}^{2}$ \\
\hline$n$ & $p=28$ & 29 & 30 & 31 & 32 & 33 & 34 & 35 & 36 & 37 & 38 & & & \\
\hline 2 & 0 & 0 & 0 & 0 & 0 & 0 & 0 & 0 & 0 & 0 & 0 & & & \\
\hline 3 & 0 & 0 & 0 & 0 & 0 & 0 & 0 & 0 & 0 & 0 & 0 & & & \\
\hline 4 & 0 & 0 & 0 & 0 & 0 & 0 & 0 & 0 & 0 & 0 & 0 & & & \\
\hline 5 & 0 & 0 & 0 & 0 & 0 & 0 & 0 & 0 & 0 & 0 & 0 & & & \\
\hline 6 & $Z^{2}$ & $\mathrm{Z}$ & $\mathbf{Z}^{2}$ & 0 & $\mathrm{Z}^{2}$ & 0 & $\mathrm{Z}$ & $Z$ & 0 & $z$ & 0 & & & \\
\hline
\end{tabular}

Proof: $A^{\alpha}-\Sigma_{\gamma} \rho_{\gamma} d g_{\gamma \alpha}=A^{\beta}-\Sigma_{\gamma} \rho_{\gamma}\left(d g_{\alpha \beta}+d g_{\gamma \alpha}\right)=A^{\beta}-\Sigma_{\gamma} \rho_{\gamma} d g_{\gamma \beta} \forall x \in U_{\alpha \beta} \neq \varnothing$. Suppose $\Xi^{0}=\xi_{0}^{0}+\cdots+\xi_{r}^{0}$ is a zigzag for $\omega_{q} \in H^{q}\left(\mathrm{SU}_{n}\right)$, where every $\xi_{j}^{0}$ is of the type

$$
\left(\xi_{j}^{0}\right)_{\alpha_{0} \cdots \alpha_{j}}=(i e)^{j} d g_{\alpha_{j} \alpha_{j-1}} \wedge \cdots \wedge d g_{\alpha_{1} \alpha_{0}} \wedge\left(\chi_{q-2 j}^{j}\right)^{\alpha_{0} / \cdots / \alpha_{j}}
$$

with $\chi_{q-2 j}^{j} \in \mathscr{H}_{q-2 j}\left(\mathrm{SU}_{n}\right)$ and $\left(\chi_{q-2 j}^{j}\right)^{\alpha}=\left(\chi_{q-2 j}^{j}\right)^{\beta-i e} d g_{\alpha \beta} \wedge\left(d \chi_{q-2(j+1)}^{j+1}\right)^{\alpha / \beta}$; let $\chi_{q-2 r}^{r}$ be global such that $\delta \xi_{r}=0, D_{r+1}\left[\omega_{q}\right]_{r+1}=0$ and $D \Xi^{0}=0$; we put $\chi_{q-2 j}^{j}=0$ for $j>r$. Since $L_{g}^{\star} \omega_{q}=\omega_{q}$ for all $g \in G_{\mathrm{en}}$, we canl choose all $\chi_{q-2 j}^{j}$ gauge invariant, otherwise use the Haar measure $\int_{G_{\mathrm{em}}} L_{g}^{\star} \chi_{q-2 j}^{j} d g$ to achieve this. Now $\Xi^{J}=\xi_{0}^{j}+\cdots+\xi_{r-j}^{j}$ with $\left(\xi_{k}^{j}\right)_{\alpha_{j} \cdots \alpha_{j+k}}$

$$
=(i e)^{k} d g_{\alpha_{j+k} \alpha_{j+k-1}} \wedge \cdots \wedge d g_{\alpha_{j+1} \alpha_{j}} \wedge\left(\chi_{q-2(j+k)}^{j+k}\right)^{\alpha_{j} / \cdots / \alpha_{j+k}}
$$

is a zigzag for $\chi_{q-2 j}^{j}$, as induction on $k$ shows. Define inductively

$$
\left.\tilde{\chi}_{q-2 r}^{r}:=\chi_{q-2 r}^{r}, \quad \tilde{\chi}_{q-2 j}^{j}:=\left(\chi_{q-2 j}^{j}\right)^{\alpha_{j}+i e d} d \sum_{\alpha_{j+1} \in A} \rho_{\alpha_{j+1}} d g_{\alpha_{j+1} \alpha_{j}} \wedge \tilde{\chi}_{q-2(j+1)}^{j+1}\right] \text {, }
$$

so

$$
\begin{aligned}
\tilde{\chi}_{q-2 j}^{j}= & \left(\chi_{q-2 j}^{j}\right)^{\alpha_{j}}+\sum_{k=j+1}^{r}(i e)^{k-j} \\
& \times d\left[\sum_{\alpha_{j+1} \in A} \rho_{\alpha_{j+1}} d g_{\alpha_{j+1} \alpha_{j}} \wedge \ldots \wedge d\left[\sum_{\alpha_{k} \in A} \rho_{\alpha_{k}} d g_{\alpha_{k} \alpha_{k-1}} \wedge\left(\chi_{q-2 k}^{k}\right)^{\alpha_{j}}\right] \ldots\right] .
\end{aligned}
$$

By the Collating formula all $\tilde{\chi}_{q-2 j}^{j}$ are global forms (restricted to $U_{\alpha_{j}}$ ). Finally $\forall U_{\alpha} \in \mathfrak{U}$ let 


$$
\begin{aligned}
& \left(\omega_{q}^{A}\right)^{\alpha}:=\left(\chi_{q}^{0}\right)^{\alpha}+\sum_{j=1}^{r} \underbrace{d\left[\text { ie } A ^ { \alpha } \wedge d \left[i e A ^ { \alpha } \wedge \ldots \wedge d \left[\text { ie } A^{\alpha} \wedge\right.\right.\right.}_{j}\left(\chi_{q-2 j}^{j}\right)^{\alpha} \ldots \\
& =\left(\chi_{q}^{0}\right)^{\alpha}+\sum_{j=1}^{r}\left(\text { ie } F^{\alpha}\right)^{j-1} \wedge d\left[i e A^{\alpha} \wedge\left(\chi_{q-2 j}^{j}\right)^{\alpha}\right] \\
& =\sum_{j=0}^{r}\left(\text { ie } F^{\alpha}\right)^{j} \wedge\left[\left(\chi_{q-2 j}^{j}\right)^{\alpha}-i e A^{\alpha} \wedge d\left(\chi_{q-2(j+1)}^{j+1}\right)^{\alpha}\right]
\end{aligned}
$$

Proposition V.15: $\left\{\left(\omega_{q}^{A}\right)^{\alpha}\right\}_{\alpha \in A}$ defines a gauge invariant global $\omega_{q}^{A} \in H^{q}\left(B\left(M, \mathrm{SU}_{n}, G_{e m}\right)\right)$ whose restriction to the fiber is $\omega_{q}=i_{x}^{\star} \omega_{q}^{A}$ for all $x \in M$.

Proof: We show that $\omega_{q}^{A}$ is global within the cohomology class of $\tilde{\chi}_{q}^{0}=f\left(\Xi^{0}\right)$ from Corollary V.12. By Lemma V.14 all $\beta^{j}:=\left(A^{\alpha_{j-1}}-\Sigma_{\alpha_{j}} \rho_{\alpha_{j}} d g_{\alpha_{j} \alpha_{j-1}}\right) \wedge \tilde{\chi}_{q-2 j}^{j}$ are global. Thus all $\hat{\chi}_{q-2 j}^{j}:=\tilde{\chi}_{q-2 j}^{j}+d \beta^{j+1}$

$$
\begin{aligned}
& =\left(\chi_{q-2 j}^{j}\right)^{\alpha_{j}+i e d\left(A^{\alpha_{j}} \wedge \tilde{\chi}_{q-2(j+1)}^{j+1}\right)}
\end{aligned}
$$

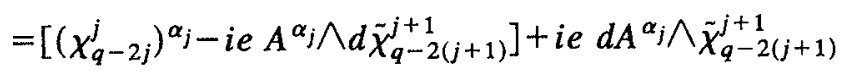

are global and differ from $\tilde{\chi}_{q-2 j}^{j}$ only by exact forms, so $d \hat{\chi}_{q-2 j}^{j}=d \tilde{\chi}_{q-2 j}^{j}$. Finally the second term on the right-hand-side of (38) is global by (18) and thus the first term is global, too. Any change of $\bar{\chi}_{q-2(j+1)}^{j+1}$ by an exact form $d \beta, \beta \in \mathscr{B}(B)$ does not change this first term at all and changes the second only by $d\left(d A^{\alpha_{j}} \wedge \beta\right)$, so again $\hat{\chi}_{q-2 j}^{j}$ is changed only by an exact form. Induction yields the desired result.

Suppose, $d^{\prime}\left(\chi_{q-2(j+1)}^{j+1}\right)=v_{q-2 j}^{j}$ from Theorem III.6, such that the zigzag exists independently of the $g_{\alpha \beta}$. Then $\left[\left(\chi_{q-2 j}^{j}\right)^{\alpha}-i e A^{\alpha} \wedge d\left(\chi_{q-2(j+1)}^{j+1}\right)^{\alpha}\right]=\left(\chi_{q-2 j}^{j}\right)^{\alpha} v$ and

$$
\left(\omega_{q}^{A}\right)^{\alpha}=\sum_{j=0}^{r}\left(\text { ie } F^{\alpha}\right)^{j} \wedge\left(\chi_{q-2 j}^{j}\right)^{\alpha} v .
$$

Adaptation to $\Gamma$ cannot mean that we look for a vertical form within the cohomology class $\left[f\left(\Xi^{0}\right)\right]$, because such a form will not exist in general. Yet we have shown that-whenever the zigzag $\Xi^{0}$ is of the given type-we find a representation $\omega_{q}^{A}$ of $\left[f\left(\Xi^{0}\right)\right]$, whose horizontal parts are mere products of the Faraday 2-form and $\omega_{q}^{A} \in H^{q}\left(B\left(M, \mathrm{SU}_{n}, G_{\mathrm{em}}\right)\right)$ independently of $M$ and the $g_{\alpha \beta}$.

Note that in our proof we did not use any special properties of $\mathrm{SU}_{n}$. Indeed, the result holds for any bundle $B\left(M, F, G_{\mathrm{em}}\right)$. Observe in addition that for the generated cohomology groups $\left\langle\omega_{q}\right\rangle \leqslant\left\langle\omega_{q}^{A}\right\rangle$ holds, i.e. $H^{q}(F, Z) \supseteq\left\langle\omega_{q}\right\rangle \cong Z$ yields $H^{q}(B, Z) \supseteq\left\langle\omega_{q}^{A}\right\rangle \cong Z$ because $i_{x}^{\star}: H^{*}(B, Z) \rightarrow H^{*}(F, Z)$ is a homomorphism. [If $z \omega_{q}^{A}=d \alpha$ with $\alpha \in \mathscr{A}_{q-1}(B)$, then $z \omega_{q}=i_{x}^{\star}\left(z \omega_{q}^{A}\right)=i_{x}^{\star} d \alpha=d\left(i_{x}^{\star} \alpha\right)$.]

Our explicit zigzags for $\omega_{2 l+1}$ yield the following corollary to Proposition V.15:

Theorem V.16: Gauge invariant generalizations of $\omega_{3}$ and $\omega_{5}$ adapted to $\Gamma$ and generating cohomology groups isomorphic to $\mathrm{Z}$, are

$$
\begin{aligned}
\left(\omega_{3}^{A}\right)^{\alpha} & =\omega_{3} v+\text { ie } F \wedge \chi_{1}^{1} v=\left[\omega_{3}^{\alpha}-3 i e A^{\alpha} \wedge\left(\rho_{2}^{Q}-\lambda_{2}^{Q}\right)\right]+3 i e F \wedge\left(\rho_{1}^{Q}+\lambda_{1}^{Q}\right) \\
\left(\omega_{5}^{A}\right)^{\alpha} & =\omega_{5} v+i e F \wedge \chi_{3}^{1} v+(i e)^{2} F \wedge F \wedge \chi_{1}^{2} v \\
& =\left[\omega_{5}^{\alpha}-5 i e A^{\alpha} \wedge\left(\rho_{4}^{Q}-\lambda_{4}^{Q}\right)\right]+5 i e F \wedge\left\{\left(\rho_{3}^{Q}+\lambda_{3}^{Q}\right)^{\alpha}\right.
\end{aligned}
$$




$$
\begin{aligned}
& \left.-2 i e A^{\alpha} \wedge \operatorname{Tr}\left[Q^{2}\left(R^{2}-L^{2}\right)+Q d U^{\dagger} \wedge Q d U\right]^{\alpha}\right\} \\
& +5(i e)^{2} F \wedge F \wedge\left[2\left(\rho_{1}^{Q^{2}}+\lambda_{1}^{Q^{2}}\right)^{\alpha}+\operatorname{Tr}\left(Q d U Q U^{\dagger}-Q U Q d U^{\dagger}\right)^{\alpha}\right]
\end{aligned}
$$

These forms coincide with the ones found by Witten ${ }^{6}$ and Kaymakcalan, Rayeev and Schechter. ${ }^{7} \omega_{3}^{A}$ serves for the locally conserved baryonic current

$$
\tilde{B}^{A}(U)=*\left(\frac{-1}{4 \pi^{2}} U^{\star} \omega_{3}^{A}\right) \in \mathscr{A}_{1}(M) \text { with } \delta \tilde{B}^{A}(U)=0
$$

whereas the integral over $\omega_{5}^{A}$ is the Wess-Zumino term for the skyrmion bundle

$$
\Gamma_{W Z}(U, A)=\frac{i N_{C}}{240 \pi^{2}} \int_{D^{5}}\left(U^{\prime}\right)^{\star} \omega_{5}^{A},
$$

completing $\Gamma(U, A)=\Gamma_{N A}(U, A)+\Gamma_{W Z}(U, A)$ with $\Gamma_{N A}(U, A)$ from (30). Nevertheless these forms are not unique in the sense that they are the only possible generalizations of type (39). As already pointed out in Ref. 7 , an additional term

$$
r(i e)^{l} F^{l} \wedge d \operatorname{Tr}\left(Q U^{\dagger} Q U\right), \quad r \in \mathbb{R},
$$

may be added to $\omega_{2 l+1}^{A}$, and this is still of the given typc, because $d \operatorname{Tr}\left(Q U^{\dagger} Q U\right)$ is global, gaugc invariant and vertical. From the mathematical point of view, one could even add any $F^{l} \wedge d \alpha$ with $\alpha=L_{\varepsilon}^{\star} \alpha \in \mathscr{A}_{0}\left(\mathrm{SU}_{n}\right)$. In order to exclude these, one needs further physical requirements like parity invariance, ${ }^{7}$ equality of the numbers of $F$ 's and $Q$ 's, etc.

\section{SUMMARY AND FURTHER COMMENTS}

We have proven that all homotopy groups of skyrmion bundles $B$ are independent of the magnetic charges of the monopoles and their locations. The same holds for the physically relevant cohomology groups $H^{3}(B, Z) \cong Z$ and $H^{5}(B, Z) \cong Z$. We have further shown that the generalizations of $\omega_{3}$ and $\omega_{5}$ in Refs. 6 and 7 are generators for these groups and thus are not only closed but also non-exact. Equation (39) gives a very compact notation of these forms and a mathematically rigorous description of this "trial-and-error adaptation to the Maxwell connection."

These forms now allow for the treatment of the monopole-induced proton decay within the skyrmion bundle. In fact, although we have proven that $\omega_{3}^{A}$ is a correct closed differential form for the locally conserved baryonic current, and although $H^{3}(B, Z) \cong Z$, the number of baryons $B^{A}(U)$ is not topologically conserved any more, whenever magnetic monopoles are present. This is due to the fact, that in contrast to the ungauged Skyrme model, the Index theorem II.4 does not apply any more. There is no possibility to compactify space to an $\mathrm{S}^{3}$, so the topological charge can vanish through the monopole singularities, as described in those scenarios of Callan and Witten, ${ }^{2}$ resp., Chemtob. ${ }^{3}$ [For the latter, his result that the proton decays independently from the initial impact parameter such that no finite reaction cross section exists, is somewhat unsatisfactory from the physicists viewpoint. We cannot decide whether his result is connected with his additional term in the baryonic current-the last one in his equation (7). The origin of this term remains unclear, in particular since it is zero anyhow.]

Generalizations of (39) to non-abelian Yang-Mills theories are possible. For example, take $G=U_{n}^{L} \times U_{n}^{R}, F=\mathrm{U}_{n}$ with $L_{\left(g_{L}, g_{R}\right)}(U)=g_{L} U g_{R}^{-1}$ and for $M$ 4-dimensional space-time (eventually with points excluded). Let $A^{\alpha}=\left(A_{L}^{\alpha}, A_{R}^{\alpha}\right)$ and $F^{\alpha}=\left(F_{L}^{\alpha}, F_{R}^{\alpha}\right) \in \mathscr{A}\left(U_{\alpha}, \mathfrak{u}_{n}^{L} \oplus \mathfrak{u}_{n}^{R}\right)$ define the connection $\Gamma$ on $P(M, G)$. Then $d U v=d U+A_{L} U-U A_{R}$, so

$$
L v=L+U^{\dagger} A_{L} U-A_{R}, \quad R v=R+A_{L}-U A_{R} U^{\dagger}
$$


and a painstaking calculation shows that the voluminous expressions for $\Gamma_{W Z}\left(U, A_{L}, A_{R}\right)$ in equation (4.18) in Ref. 7, resp., equation (24) in Ref. 6 are equal to the integral over

$$
\omega_{5} v+\chi_{3}^{1} v(F)+\chi_{1}^{2} v(F, F)
$$

where, with the projections $\pi^{L / R}: \mathfrak{g}=\mathfrak{u}_{n}^{L} \oplus \mathfrak{u}_{n}^{R} \rightarrow \mathfrak{u}_{n}^{L / R}, \chi_{5-2 l}^{l} \in \mathscr{f}_{5-2 l}\left(\mathrm{U}_{n}, \operatorname{Hom}(\otimes \mathfrak{g}, \mathrm{C})\right)$ :

$$
\chi_{3}^{1}=-5 \operatorname{Tr}\left(R^{3} \pi^{L}+L^{3} \pi^{R}\right)
$$

i.e.

$$
\chi_{3}^{1}(F)=-5 \operatorname{Tr}\left(R^{3} F_{L}+L^{3} F_{R}\right)
$$

and

$$
\chi_{1}^{2}=10 \operatorname{Tr}\left(R \pi^{L} \pi^{L}+L \pi^{R} \pi^{R}\right)+5 \operatorname{Tr}\left(d U \pi^{R} U^{\dagger} \pi^{L}-d\left(U^{\dagger}\right) \pi^{L} U \pi^{R}\right) .
$$

Analogously to the skyrmion case, one may add a term

$$
r\left[d \operatorname{Tr}\left(\pi^{L} U \pi^{R} U^{\dagger}\right) v\right](F, F)=r d \operatorname{Tr}\left(F_{L} U F_{R} U^{\dagger}\right), r \subset \mathrm{C},
$$

or exclude it by parity invariance.

\section{ACKNOWLEDGEMENTS}

The author is very grateful to Prof. Dr. F. Beck and Prof. Dr. K. H. Hofmann for initializing and supporting this research, resp., giving him an introduction to spectral sequences and for numerous fruitful discussions. The author is supported by Studienstiftung des deutschen Volkes.

${ }^{1}$ T. H. R. Skyrme, "A unified field theory of mesons and baryons," Nucl. Phys. 31, 556-569 (1962).

${ }^{2}$ C. E. Callan and E. Witten, "Monopole catalysis of skyrmion decay," Nucl. Phys. B 239, 161-176 (1984).

${ }^{3}$ M. Chemtob, "Cross section of monopole-induced skyrmion decay," Phys. Rev. D 39, 2013-2031 (1989).

${ }^{4}$ R. Bott, "The stable homotopy of the classical groups," Ann. Math. 70, 313-337 (1959).

${ }^{5}$ T. Eguchi, P. B. Gilkey, and A. J. Hanson, "Gravitation, gauge theories and differential geometry," Phys. Rep. 66, 213-393 (1980).

${ }^{6}$ E. Witten, "Global aspects of current algebra," Nucl. Phys. B 223, 422-432 (1983).

'Ö. Kaymakcalan, S. Rajeev, and J. Schechter, "Non-abelian anomaly and vector-meson decays," Phys. Rev. D 30, 594-602 (1984).

${ }^{8}$ N. K. Pak and P. Rossi, "Gauged Goldstone boson effective action from direct integration of Bardeen anomaly," Nucl. Phys. B 250, 279-294 (1985).

${ }^{9} \mathrm{C}$. Gross, "Generating functions of $\pi_{2 n-1}\left(\mathrm{SU}_{n}\right)$," Seminar Sophus Lie 3, 89-105 (1993).

${ }^{10}$ R. Bott and R. Seeley, "Some remarks on the paper of callias," Commun. Math. Phys. 62, 235-245 (1978).

${ }^{11} \mathrm{~J}$. Wess and B. Zumino, "Consequences of anomalous Ward identities," Phys. Lett. B 37, 95-97 (1971).

${ }^{12}$ I. Zahed and G. E. Brown, "The Skyrme model," Phys. Rep. 142, 1-102 (1986).

${ }^{13}$ W. A. Poor, Differential Geometric Structures (McGraw-Hill, New York, 1981).

${ }^{14}$ R. Abraham, J. E. Marsden, and T. Ratiu, Manifolds, Tensor Analysis, and Applications (Addison-Wesley, Reading, MA, 1983).

${ }^{15} \mathrm{C}$. Nash and S. Sen, Topology and Geometry for Physicists (Academic, London, 1983).

${ }^{16}$ N. Steenrod, The Topology of Fibre Bundle's (Princeton University Press, Princeton, NJ, 1951).

${ }^{17}$ S. Kobayashi and K. Nomizu, Foundations of Differential Geometry (Wiley, New York, 1963), Vol I.

${ }^{18}$ R. Bott and L. W. Tu, Differential Forms in Algebraic Topology (Springer-Verlag, New York, 1982).

${ }^{19}$ Encyclopedic Dictionary of Mathematics, 2nd ed. (MIT Press, Cambridge, MA, 1987), Vol. IV, Appendices and Indexes.

${ }^{20}$ E. H. Spanier, Algebraic Topology (McGraw-Hill, New York, 1966). 